Proc. Indian Acad. Sci. (Engg. Sci.) Vol. 4, Pt. 3, September 1981, pp. 315-345 @ Printed in India.

\title{
Inviscid transonic flow field analysis
}

\author{
T C LIN \& JIA ZHENXUE \\ Institute of Mechanics, Academia Sinica, Beijing, People's Republic of China
}

MS received 26 August 1981.

\begin{abstract}
This paper describes a method for analysing inviscid transonic flow. This method is based on the fact that the angle made by the streamline of the transonic flow and of the corresponding incompressible flow is usually small. By using curvilinear coordinates, the differential equation of the stream function of an inviscid compressible flow is simplified and a general solution of the equation obtained.

As examples of the method, transonic solutions are given for flow through twodimensional and axisymmetric Laval nozzles of different throat wall radii together with sonic lines and iso-Mach lines. To determine the discharge coefficients of Laval nozzles, an integral relation is developed. The general behaviour of the transonic flow in the throat region is presented, and the effect of the mass discharge on the Mach number distribution in the nozzle analysed. The effects of the ratio of the specific heats on the characteristics of the flow in the throat region are discussed. For transonic flow around a circular cylinder and a sphere, sonic lines and iso-Mach lines are presented for free-stream Mach number varying from the subcritical to the supercritical, including a free-stream Mach number of one.

Part of the results obtained are compared with those available in current literature. For the two-dimensional hyperbolic Laval nozzles, the iso-Mach lines are compared with those given by Cherry (1959) and Serra (1972). For. axisymmetric Laval nozzles, the discharge coefficient and the Mach number at the throat section for various throat wall radii are compared with those given by Sauer (1944), Hall (1962), Kliegel \& Levine (1969), and Klopfer \& Holt (1975). The theoretical discharge coefficients are compared with the experimental results by Back et al (1975), Durham (1955), Norton \& Shelton (1969) etc. For the transonic flow around a circular cylinder, the iso-Mach lines are compared with Cherry's exact solution for the quasi-circular cylinder for $M_{\infty}$ equal to 0.51 . The Mach number distributions on the surface of the circular cylinder are compared with those given by Imai (1941) for $M_{\infty}$ equal to 0.4 , by Cherry (1947) for $M_{\infty}$ equal to 0.51 , by Dorodnicyn (1956) for $M_{\infty}$ equal to 1 , and by Hafez, South \& Murman (1979) for $M_{\infty}$ equal to 0.51 .

The present method has a much wider scope of application, requires simpler computation and gives results with good accuracy. It is being used to analyse supercritical wings and cascades, and we expect to extend its application to the field of transonic unsteady flow.
\end{abstract}

Keywords. Transonic flow; Laval nozzles; flow field computations; flow past circular cylinder.

\section{Introduction}

A century has passed since De Laval (1883) invented the convergent-divergent nozzle, and Reynolds (1886) developed the one-dimensional subsonic-supersonic nozzle flow theory. An investigation of the representative works of the past century shows that the developments in this field may be roughly divided into three periods (see figure 1).

For the first sixty years, i.e., from the early 1880 s to the late 1930 s, the problem of transonic flow was for the first time proposcd and some fundamental investigations

Proc. -3 
essential to the founding of this branch of science were made. Notable contributors of this period include Chaplygin (1904), Meyer (1908), Tricomi (1923), Taylor (1930), and Görtler (1939). The hodograph method (Chaplygin 1904), the series expansion method (Meyer 1908; Taylor 1930; Görtler 1939), ard the simplified transonic flow equation (Tricomi 1923) were introduced, and a number of transonic flow solutions obtained. However, the development was slow relative to the time span involved, as is the case with most of the virgin fields of study.

In the early forties, the speed of the aeroplane increased rapidly and approached that of sound. Owing to the so-called 'sonic barrier', growing attention was accorded to transonic flow research. Many aerodynamicists and mathematicians took part in this work, and remarkable progress was made. However, with the overcoming of the 'sonic barrier' through engine thrust, the next 15 years witnessed an obvious decrease in the research, followed by a state of low activity. These 25 years might be considered as the second period of transonic flow research. This notable upsurge and subsequent decrease in the activities of research is graphically shown in figure 1.

In this period the analytic methods of the early days, such as the hodograph method (Von Ringleb 1940; Frankl 1945; Lightill 1947; Goldstein et al 1948; Cherry 1947, 1949a, b, 1950, 1959; Tsien \& Kuo 1946, Kuo 1948, 1951; Tomotika \& Tamada 1950) and the series expansion method (Sauer 1944) were taken over and further developed. In addition, a number of distinct approaches were proposed, such as the small parameter expansion method (Imai 1941; Simasalsi 1956; Hall 1962; Kliegel \& Levine 1969), the relaxation method (Green \& Southwell 1944; Emmons 1946, 1948) the law of transonic similarity (Von Karman 1947; Lin et al 1948), the integral equation method (Oswatitsch 1950; Gullstrand 1951a, b; Spreiter \& Alksne 1955), the time dependent method (Von Neuman \& Richtmyer 1950; Lax \& Wendroff 1964; Crocco 1965; Moretti \& Abbett 1966), and the strip integration method (Dorodnitsyn 1956; Chushkin 1957; Holt 1962). All this not only improved and enriched the analytic methods of the earlier days, but also provided a theoretical basis for the numerical methods characteristic of the period to come.

In the fifteen years since mid-sixties, the pendulum seems to have swung back. The problem of transonic flow has once again claimed the attention of many researchers.

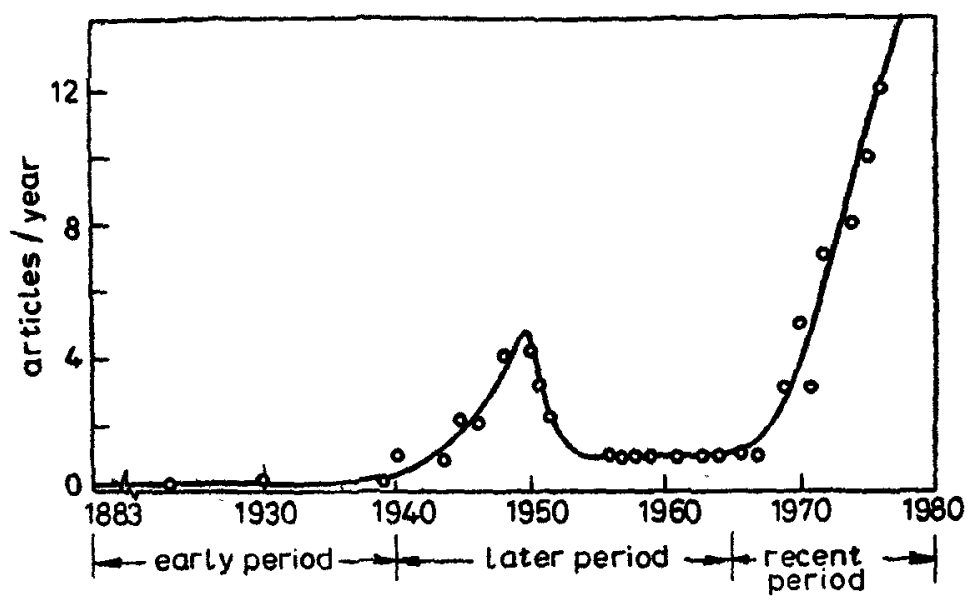

Figure 1. Development of transonic studies. 
This second upsurge in transonic research has been necessitated by the urgent demand in air tranisport and space exploration for greater payloads, better cruising performance of large transport and shuttles, and higher efficiencies of jet engines, turbo-machines, and cascades. Flights in the transonic 'realm, once deliberately avoided, have become the object of intensive study with their inherent advantages being maximally exploited. This trend in research has been further accelerated by the recent energy crisis in the industrial world.

One of the dominant features of transonic flow study during this period is the rapid development and extensive application of numerical methods. Many of the transonic flow problems that used to be tackled with wind tunnels, are being solved with the aid of digital computers. The main numerical methods being developed are the hodograph method (Nieuwland 1967; Boerstoel \& Uijlennoel 1970; Boerstoel 1974; Takanashi 1973; Garabedian \& Lieberstein 1958; Swenson 1968; Korn 1969; Garabedian \& Korn 1971; Bauer et al 1975; Sobieczky 1972), the relaxation method (Murman \& Cole 1971; Murman 1973; Krupp 1971; Krupp \& Murman 1972; Jameson 1971, 1974, 1975a, b; Hofez \& Cheng 1977a, b; Cheng \& Hafez 1976), the time-dependent method (Magnus et al 1968; Magnus \& Yoshihara 1970; Moretti 1969, 1970; Grossman \& Moretti 1973; Serra 1972; Cline 1974), the integral equation method (Nфrstrud 1973; Hansen 1975; Nixon 1975, 1978; Chakrabarty 1978), the integral relation method (Holt \& Masson 1971; Gross \& Holt 1975; Klopfer \& Holt 1975), and the finite element method (Chan \& Brashears 1974; Shen 1975, 1977; Norrie \& de Vries 1975; Chan et al 1975). These methods, though inherited from the previous period, have taken on a new character by the introduction of numerical procedures. This period, which might conveniently be called the third stage of transonic research, has made a promising start and has up to now shown no signs of abatement.

In fact, transonic flow research is currently in a state of fast development, with new findings appearing in rapid succession. However, along with the fruitful results achieved, a series of new problem, have also emerged. First of all, as the general line of attack has been the search for adequate computing schemes, based on the theories of the previous period, greater effort has been made from the viewpoint of numerical computation than from that of the physical characteristics of the flow. Besides, for the purpose of obtaining convergent solutions some man-made factors, such as artificial viscosity, are being introduced in numerical computation, and this tends to affect the accuracy of the results. Moreover, while notable success has been made with respect to thin wings, relatively little has been achieved for thick bodies. For example, in respect of transonic flow about a cylinder, the sonic lines and the iso-Mach lines for different free-stream Mach numbers, especially for free-stream Mach number equal to 1 , have not been made clear, in spite of the many interesting papers published on the topic. In the case of transonic flow about a sphere, the situation also calls for further study. Another feature characteristic of current research is by and large the use of the potential function rather than the stream function as a means of solving transonic flow problems. One of the main reasons for this prevailing preference has been the difficulties in solving the many-valued function with the stream-function approach. But as a matter of fact the stream-function, with its more obvious physical property, may, when properly used, well prove to be a more efficient approach both in theoretical analysis and in numerical computation. 
In view of all this, the present paper proposes to adopt the stream-function approach for the solution of transonic flow problems. A method has accordingly been developed for analysing transonic flow, based indeed on the simple but neglected physical fact that the angle made by the streamline of the transonic flow and that of the corresponding incompressible flow is usually small. By using curvilinear coordinates, the differential equation of the stream function of a compressible flow can be simplified and a general solution obtained.

As examples of the application of the method, a number of specific transonic flow problems are analysed and solutions, including sonic lines and iso-Mach lines of the flow fields, are given. In the case of internal flow, the flow fields through twodimensional and axisymmetric Laval nozzles of different contraction ratios and different throat wall radii are analysed for various mass flow rates. In the case of external flow, the flow fields about a circular cylinder and about a sphere are analysed for free stream Mach number $M_{\infty}$ varying from the subcritical to the supercritical including $M_{\infty}$ equal to 1 .

Some of the results obtained by the present method are compared with those available in current literature. It will be found that the present method has a much wider scope of application, requires far simpler computation and gives results of good accuracy.

The present method is being used to analyse supercritical wings and cascades, and we expert to extend its use to the study of transonic unsteady flow.

\section{The basic equation and the solution}

We shall confine our discussion to the study of the steady-state, irrotational, isentropic, two-dimensional or axisymmetric flow of an ideal gas. The non-dimensionalized quantities are signified by a bar placed over the corresponding symbols:

$$
\left.\begin{array}{ll}
\bar{x}=x / b & \bar{p}=p / p_{*} \\
\bar{y}=y / b & \rho=\rho / \rho_{*} \\
\bar{z}=\bar{x}+i \bar{y} & \bar{T}=T / T_{*} \\
\bar{u}=u / a_{*} & \bar{\phi}=\phi / a_{*} b(\pi b)^{\epsilon} \\
\bar{v}=v / a_{*} & \bar{\psi}=\psi / a_{*} \rho_{*}(\pi b)^{\epsilon} \\
\bar{q}=q / a_{*} & M=\bar{q} / \bar{a} \\
\bar{a}=a / a_{*} & \bar{r}=r / b
\end{array}\right\}
$$

Here $b$ denotes characteristic length and the asterisk denotes sonic condition. As only non-dimensional quantities are to be used in this paper, the bar over the symbol is invariably left out. 
For the two-dimensional or axisymmetric irrotational flow of ideal gas, the mass, momentum and energy conservation relations can be respectively represented in orthogonal curvilinear coordinates (Tsien 1958) as follows:

$$
\left.\begin{array}{l}
\left(y^{\epsilon} H_{2} \rho u\right)_{\xi}+\left(y^{\epsilon} H_{1} \rho v\right)_{\eta}=0, \\
\left(H_{2} v\right)_{\xi}-\left(H_{1} u\right)_{\eta}=0, \\
T=\frac{\gamma+1}{2}-\frac{\gamma+1}{2} q^{2}, \\
H_{1}=\left(x_{\xi}^{2}+y_{\xi}^{2}\right)^{1 / 2}, H_{2}=\left(x_{\eta}^{2}+y_{\eta}^{2}\right)^{1 / 2}
\end{array}\right\}
$$

In the above equations $H_{1}$ and $H_{2}$ are the inverse of the Lamé coefficients (1934) for the transformation between the orthogonal curvilinear coordinates $(\xi, \eta, \theta)$ and the orthogonal coordinates $(x, y, \theta) ; u$ and $v$ are the velocity components along $\xi, \eta ; \rho$ and $T$ express respectively the density and absolute temperature of the gas; $\epsilon=0$ denotes plane motion, and $\epsilon=1$ denotes axisymmetric motion.

The equation of state of a perfect gas, the adiabatic flow relation and the relation between temperature $T$ and sonic velocity $a$ under adiabatic conditions, when expressed in the non-dimensionalized variables chosen for this paper, may be written as follows:

$$
p=\rho T, \quad p=\rho^{\gamma}, \quad a=T^{1 / 2}
$$

From (2) and (3), the relations between either density $\rho$ or Mach number $M$ and the velocity $q$ are obtained respectively as

$$
\left.\begin{array}{l}
\rho=\left(\frac{\gamma+1}{2}-\frac{\gamma-1}{2} q^{2}\right)^{\frac{1}{\gamma-1}}, \\
M=q /\left(\frac{\gamma+1}{2}-\frac{\gamma-1}{2} q^{2}\right)^{1 / 2} \cdot
\end{array}\right\}
$$

From equations $(2 \mathrm{a}, \mathrm{b}) *$ one can define stream function $\psi$ and potential function $\phi$ as follows:

$$
\left.\begin{array}{ll}
\psi_{\xi}=-\rho y^{\epsilon} H_{1} v, & \psi_{\eta}=\rho v^{\epsilon} H_{2} v, \\
\phi_{\xi}=H_{1} u, & \phi_{\eta}=H_{2} v .
\end{array}\right\}
$$

Using (3) and (4) and the definition given in (5), equation (2) gives the stream-function equation for two-dimensional or axisymmetric flow as

*The letters $a, b$ etc. attached to the Arabic number indicate the sequential order of the formulas each equation. 


$$
\begin{aligned}
& \left(y^{2 \epsilon} H_{2}^{2} a^{2} \rho^{2}-\psi_{\eta}^{2}\right) \psi_{\xi \xi}+2 \psi_{\xi} \psi_{\eta} \psi_{\xi \eta}+\left(y^{2 \epsilon} H_{1}^{2} a^{2} \rho^{2}-\psi_{\xi}^{2}\right) \psi_{\eta \eta} \\
& \quad+H_{1} \psi_{\eta}^{2}\left[\frac{H_{2}}{H_{1}}\left(\frac{1}{y^{\epsilon} H_{2}}\right)_{\xi} \psi_{\xi}+\frac{H_{1}}{H_{2}}\left(\frac{1}{y^{\epsilon} H_{2}}\right)_{\eta} \psi_{\eta}\right] y^{\epsilon} \\
& \quad+H_{2} \psi_{\xi}^{2}\left[\frac{H_{2}}{H_{1}}\left(\frac{1}{y^{\epsilon} H_{1}}\right)_{\xi} \psi_{\xi}+\frac{H_{1}}{H_{2}}\left(\frac{1}{y^{\epsilon} H_{1}}\right)_{\eta} \psi_{\eta}\right] y^{\epsilon} \\
& \quad-\left(\frac{H_{2}}{H_{1}} \psi_{\xi}^{2}+\frac{H_{1}}{H_{2}} \psi_{\eta}^{2}-y^{2 \epsilon} H_{1} H_{2} a^{2} \rho^{2}\right)\left[\left(\frac{H_{2}}{y^{\epsilon} H_{1}}\right)_{\xi} \psi_{\xi}\right. \\
& \left.\quad+\left(\frac{H_{1}}{y^{\epsilon} H_{2}}\right)_{\eta} \psi_{\eta}\right] \eta^{\epsilon}=0 .
\end{aligned}
$$

When $H_{1}=H_{2}=H$, this equation can be simplified. For axisymmetric flow, $\epsilon=1$, equation (6) becomes

$$
\begin{aligned}
& \left(y^{2} H^{2} a^{2} \rho^{2}-\psi_{\eta}^{2}\right) \psi_{\xi \xi}+2 \psi_{\xi} \psi_{\eta} \psi_{\xi \eta}+\left(y^{2} H^{2} a^{2} \rho^{2}-\psi_{\xi}^{2}\right) \psi_{\eta \eta} \\
& -\frac{1}{H}\left(\psi_{\xi}^{2}+\psi_{\eta}^{2}\right)\left(H_{\xi} \psi_{\xi}+H_{\eta} \psi_{\eta}\right)-y H^{3} a^{2} \rho^{2}\left(y_{\xi} \psi_{\xi}+y_{\eta} \psi_{\eta}\right)=0
\end{aligned}
$$

For two-dimensional flow $\epsilon=0$, equation (6) becomes

$$
\begin{gathered}
\left(H^{2} a^{2} \rho^{2}-\psi_{\eta}^{2}\right) \psi_{\xi \xi}+2 \psi_{\xi} \psi_{\eta} \psi_{\xi \eta}+\left(H^{2} a^{2} \rho^{2}-\psi_{\xi}^{2}\right) \psi_{\eta \eta} \\
-\frac{1}{H}\left(\psi_{\xi}^{2}+\psi_{\eta}^{2}\right)\left(H_{\xi} \psi_{\xi}+H_{\eta} \psi_{\eta}\right)=0 .
\end{gathered}
$$

When $H_{1}=H_{2}=H$ one can utilize the conformal relations between the two coordinate systems $(x, y)$ and $(\xi, \eta)$. According to the complex variable theory (Riemann 1851) the following relations are obtained:

$$
\left.\begin{array}{l}
z=f(\zeta), \\
z=x+i y, \\
\zeta=\xi+i \eta, \\
H=\left|\frac{d z}{d \zeta}\right| \cdot
\end{array}\right\}
$$

where $f(\zeta)$ is an analytic functions of $\zeta$.

Equation (6) is valid for any orthogonal curvilinear coordinated system. It is obvious that to solve this equation mathematically is very difficult, especially under transonic conditions. For this reason, fluid dynamicists have often made use of the physical characteristics of the flow, simplified the flow equation through an order-ofmagnitude analysis, and obtained approximate solutions with a certain degree of accuracy. For instance, Oswatitsch (1950) and Von Karman (1947), by making such 
an analysis for transonic flow in rectangular coordinates, gave the small disturbance equation as

$$
\phi_{x} \phi_{x x}-\frac{a_{*}}{\gamma+1} \phi_{y y}=0 .
$$

By employing a similar method in the velocity plane, Tricomi (1923) obtained a simplified transonic equation

$$
\psi_{q} \psi_{q q}-(\gamma+1) \psi_{\theta \theta}=0
$$

Their efforts have contributed greatly to the solution of the transonic small disturbance problem. But as regards the flow in a nozzle with a comparatively large throat curvature (figure 2), or the flow past a compartively thick body (figure 3), the simplified transonic flow equations described above are no longer valid. Some authors did solve the transonic problem by making one of the curvilinear coordinates coincide with the body surface. This, of course, helps tackle the boundary conditions in a convenient manner. But it fails to give a satisfactory and definite order-of-magnitude relation (figure $3 \mathrm{a}$ ) between the velocity components in the whole flow field, and cannot provide a simplified flow equation.

It was against this background that we began to examine the problem of transonic flow through a nozzle (figure 2) and the flow past a circular cylinder (figure 3 ) and we note the important fact that the angle made by the stream lines of a compressible flow and those of the corresponding incompressible flow is usually small. In other

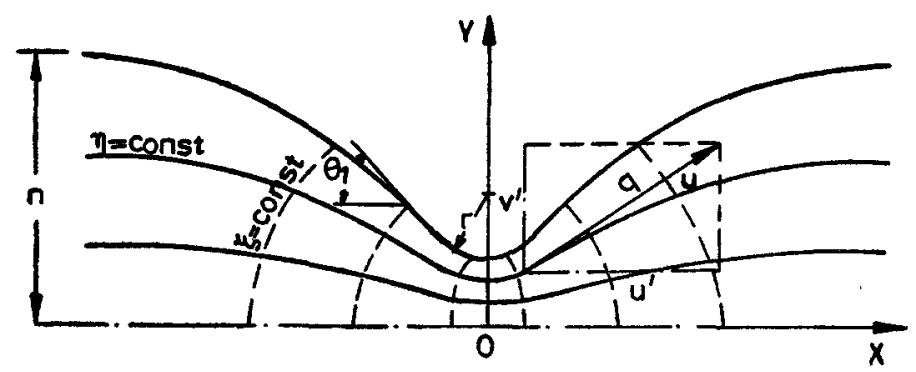

Figure 2. Laval-nozzle coordinates and the flow pattern.

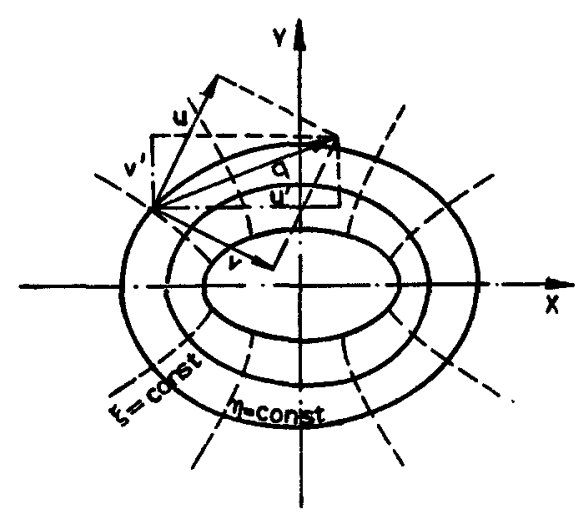

Figure 3(a). Elliptical coordinates.

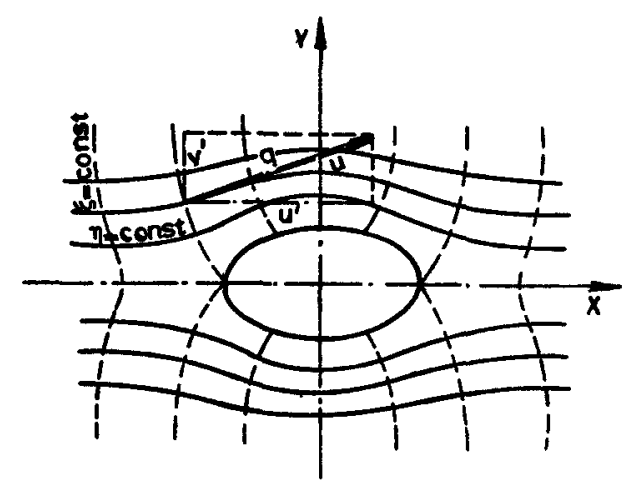

Figure 3(b). Incompressible-flow coordinates about an ellipse. 
words, with $u$ as the velocity component along $\eta$ and $v$ the one along $\xi, u$ is far greater than $v$. Therefore, selecting the equipotential lines and the streamlines of an incompressible flow as the $(\xi, \eta)$ coordinates, (6) can be successfully simplified. Assuming $u \gg v$, and using (5) we obtain $\left|\psi_{\eta}\right| \gg\left|\psi_{\xi}\right|$; by omitting $\psi_{\xi}$ and its derivatives from (6), we obtain the simplified stream function equation,

$$
y^{2 \epsilon} H_{1} a^{2} \rho^{2} \psi_{\eta \eta}-\frac{H_{1 \eta}}{y^{\epsilon} H_{2}^{2}} \psi_{\eta}^{3}+y^{2 \epsilon} H_{2} a^{2} \rho^{2}\left(\frac{H_{1}}{y^{\epsilon} H_{2}}\right)_{\eta} \psi_{\eta}=0
$$

This equation is a nonlinear ordinary differential equation in $\eta$, with $\xi$ as a parameter. Utilizing (4a) after differentiating it with respect to $\eta$ and substituting it in (10), we eliminate $\psi_{\eta \eta}$ and obtain the first integral of (10),

$$
\psi_{\eta}=y^{\epsilon}(\xi, \eta) \rho(\xi, \eta) g(\xi) \frac{H_{2}(\xi, \eta)}{H_{1}(\xi, \eta)} \text {. }
$$

Here $y^{\epsilon}(\xi, \eta)$ is a known function whereas $g(\xi)$ is a function to be determined. For flow through a nozzle, $g(\xi)$ is related to the mass discharge; for flow around a body, $g(\xi)$ is related to the free-stream Mach number. For convenience, $g(\xi)$ can be designated as a 'discharge function' with $\eta=\eta_{1}$ as the null stream line i.e. $\psi\left(\xi, \eta_{1}\right)=0$. Substituting (4a) in (11), one obtains a general solution for $\psi(\xi, \eta)$ :

$$
\begin{aligned}
& \psi(\xi, \eta)=\left(\frac{\gamma+1}{2}\right)^{\frac{1}{\gamma-1}} g(\xi) \int_{\eta_{1}}^{\eta}\left[1-\frac{\gamma-1}{\gamma+1} \frac{g^{2}(\xi)}{H_{1}^{2}(\xi, \eta)}\right]^{\frac{1}{\gamma-1}} \\
& \quad \times \frac{H_{2}(\xi, \eta)}{H_{1}(\xi, \eta)} y^{\epsilon}(\xi, \eta) d \eta .
\end{aligned}
$$

Here $H_{1}(\xi, \eta), H_{2}(\xi, \eta)$ and $y(\xi, \eta)$ are determined once the coordinate transformation is chosen, and only $g(\xi)$ is to be sought. The determination of $g(\xi)$ will be discussed later in this paper with reference to specific examples.

With $g(\xi)$ determined, the corresponding transonic flow field is established. In accordance with the stream function defined by (5), one obtains the velocity distribution in the flow field as

$$
u(\xi, \eta)=\frac{g(\xi)}{H_{1}(\xi, \eta)}, \quad v(\xi, \eta)=\frac{-\psi^{\xi}}{p H_{1}(\xi, \eta) y^{\xi}(\xi, \eta)} .
$$

\section{Internal flow}

In what follows, the above method is applied to transonic flow in a Laval nozzle. Given the nozzle coordinates, we analyse the maximum mass discharge and study the transition from the subsonic through the sonic to the supersonic regime in the throat region. A series of two-dimensional and axisymmetric sample flow fields in the throat region are presented, and the influence of the specific heat ratio $\gamma$ is obtained. 


\subsection{Nozzle coordinates}

In accordance with the incompressible potential flow and the complex variable theory, by selecting a slitted symmetrical biconvex wing section in the velocity plane, one obtains a symmetric nozzle in the physical plane. The mapping relation $f(\zeta)$ (Lin Tong $\mathrm{Ji}$ and Jia Zhen Hue 1978) between plane $z$ and plane $\zeta$ is given as

$$
z=\frac{n}{\eta_{b}}\left\{\zeta-(1-\sigma) \operatorname{coth} \lambda \ln \left[\frac{\cosh \frac{1}{2}(\lambda+\zeta)}{\cosh \frac{1}{2}(\lambda-\zeta)}\right]\right\} .
$$

In this formula, $\lambda, \sigma, \eta_{b}$ are nozzle shape factors and $n$ is the nozzles contraction ratio. By separating the real and imaginary parts of (14), we obtain the relation between the $(x, y)$ and the $(\xi, \eta)$ coordinates as

$$
\begin{aligned}
& x(\xi, \eta)=\frac{n}{\eta_{b}}\left\{\xi-\frac{1}{2}(1-\sigma) \operatorname{coth} \lambda \ln \left[\frac{\cosh (\lambda+\xi)+\cos \eta}{\cosh (\lambda-\xi)+\cos \eta}\right)\right\} \\
& y(\xi, \eta)=\frac{n}{\eta_{b}}\left[\eta-(1-\sigma) \operatorname{coth} \lambda \tan ^{-1}\left(\frac{\sinh \lambda \sin \eta}{\cosh \xi+\cosh \lambda \cos \gamma_{l}}\right)\right] .
\end{aligned}
$$

By selecting corresponding shape factors $\lambda, \sigma, \eta_{b}$ according to the values of the throat wall radius $r$, contraction ratio $n$ and contraction angle $\theta_{1}$ of the nozzle, one obtains the required profile of the nozzle.

\subsection{Maximum discharge}

For the flow through a nozzle, the maximum discharge is one of the most significant parameters. It is essential in determining the transition of the flow field from the subsonic to the supersonic region in the throat. No satisfactory answer, however, has been given to the problem involved. As a solution to this problem an integral relation equation for determining the maximum discharge is presented below.

With the streamlines and equipotential lines of the corresponding incompressible flow as the coordinates, the boundary conditions can be expressed as follows:

$$
\left.\begin{array}{ll}
\eta=\eta_{1}: & \psi\left(\xi, \eta_{1}\right)=0 \\
\eta=\eta_{b}: & \psi\left(\xi, \eta_{b}\right)=\psi_{b}
\end{array}\right\} .
$$

In these equations, $\psi_{b}$ is the discharge through the nozzle; its value cannot be arbitrarily selected. For a given nozzle there exists a maximum value of $\psi_{b}$, namely the maximum discharge.

Given the throat section coordinates $\xi=\xi_{*}$ from equations (12) and (16), the maximum discharge $\psi_{s}$ through the throat area is obtained as (Lin Tong $\mathrm{Ji} \&$ Jiazhen Xue 1978)

$$
\begin{aligned}
\psi_{s}= & \left(\frac{\gamma+1}{2}\right)^{\frac{1}{\gamma-1}} g_{s} \int_{\eta_{1}}^{\eta_{b}}\left[1-\frac{\gamma-1}{\gamma+1} \frac{g_{s}^{2}}{H_{1}^{2}\left(\xi_{*}, \eta\right)}\right] \frac{1}{\gamma-1} \\
& \times \frac{H_{2}\left(\xi_{*}, \eta\right)}{H_{1}\left(\xi_{*}, \eta\right)} y^{\in}\left(\xi_{*}, \eta\right) d \eta
\end{aligned}
$$


in which $g_{s}$ satisfies the relation

$$
\begin{aligned}
\int_{\eta_{1}}^{\eta_{b}} & {\left[1-\frac{g_{s}^{2}}{H_{1}^{2}\left(\xi_{*}, \eta\right)}\right]\left[1-\frac{\gamma-1}{\gamma+1} \frac{g_{s}^{2}}{H_{1}^{2}\left(\xi_{*}, \eta\right)}\right]^{\frac{2-\gamma}{\gamma-1}} } \\
& \times \frac{H_{2}\left(\xi_{*}, \eta\right)}{H_{1}\left(\xi_{*}, \eta\right)} y^{\epsilon}\left(\xi_{*}, \eta\right) d \eta=0 .
\end{aligned}
$$

The roots of equation (17), $g_{s}$, are multi-valued, the smallest of which corresponds to the maximum discharge $\psi_{s}$. For the flow through the nozzle, $\psi_{b}$ cannot be greater than $\psi_{s}$. For a given value of $\psi_{b}$, from the boundary condition (16), one obtains the integral relation that determines the discharge function $g(\xi)$ as follows:

$$
\begin{aligned}
\psi_{b}= & \left(\frac{\gamma+1}{2}\right)^{\frac{1}{\gamma-1}} g(\xi) \int_{\eta_{1}}^{\eta_{b}}\left[1-\frac{\gamma-1}{\gamma+1} \frac{g^{2}(\xi)}{H_{1}^{2}\left(\xi ;{ }^{*} \eta\right)}\right]^{\frac{1}{\gamma-1}} \\
& \times \frac{H_{2}(\xi, \eta)}{H_{1}(\xi, \eta)} y^{\epsilon-}(\xi, \eta) d \eta .
\end{aligned}
$$

Equation (18) is an integral relation equation for $g(\xi)$ with $\xi$ as a variable parameter; for a given value of $\xi, g(\xi)$ is multivalued. In order to ensure the continuity and the non-occurrence of a negative density in the solution, $g(\xi)$ has to be selected as follows: for subsonic flow in the nozzle, $\psi_{b}<\psi_{s}$, the smallest positive root of $g(\xi)$ should be taken for the entire range of $\xi$; for supersonic flow through the nozzle, $\psi_{b}=\psi_{s}$, the selection of $g(\xi)$ is dependent upon the range of $\xi$. When $\xi<\xi_{*}$, the smallest positive root of $g(\xi)$ should be taken, and this corresponds to the flow through the contracted portion. When $\xi=\xi_{*}$, the smallest positive repeated root should be taken, which corresponds to the flow in the throat section. When $\xi>\xi_{*}$, the second positive root of $g(\xi)$ should be taken, which corresponds to the flow through the expanded portion. Figure 4 gives the variation of $g(\xi)$ with $\xi$ for various portions of the nozzle.

\subsection{Transition of the nozzle throat flow field}

For a given nozzle, there exists a maximum discharge. When the inlet discharge is smaller than the maximum discharge, i.e., the discharge coefficient (factor) $k=\psi_{b} / \psi_{s}$ is smaller than 1, the flow through the nozzle is of a subsonic symmetric type. When $k=1$, the flow is of a supersonic type. How the flow field in the throat region of a nozzle changes from the subsonic symmetric type to the supersonic type is one of the most significant problems of nozzle flow field investigations. Tomotika \& Tamada (1950) applied the hodograph method to an artificial gas and explained qualitatively the relationship between the flow field transition and the discharge in the throat region. In this section the same problem is discussed quantitatively, and with reference to an ideal rather than arificial gas.

In accordance with the general solution of nozzle flow given above, proper selections can be made for the contraction ratio $n$ of the nozzle, the maximum angle $\theta_{1}$ of inclination of the wall surface, and the radius of curvature $r$ of the throat wall. Our 

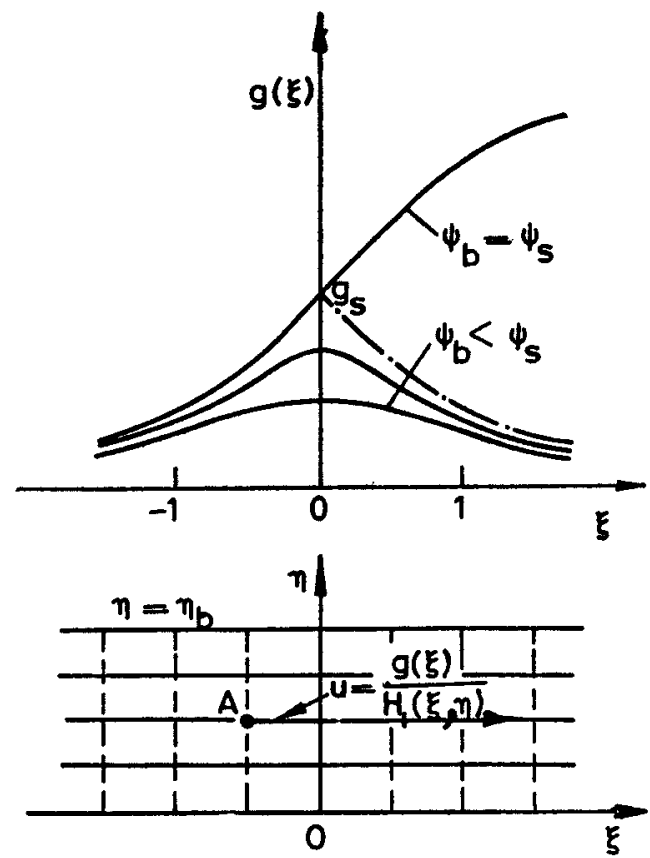

Figure 4. The "discharge correlation function" $g(\xi)$.

analysis demonstrates that the variation in the value of $\gamma$, the ratio of specific heats, hardly affects the flow field in the throat region. In view of the fact that when $\gamma$ equals 2 , the analytical expressions for the flow fields in the throat assume a simpler form and that water table tests also yield a flow condition with $\gamma=2$, one can more conveniently explain the general properties of the throat flow field transition by making $\gamma=2$. When $\gamma=2$, the analytical solution (Lin Tong Ji \& Jiazhen Xue 1978) of the throat flow field is

$$
\begin{aligned}
& \psi(\xi, \eta)=\frac{3}{2} \eta g(\xi)-\frac{1}{2} E_{1}(\xi, \eta) g^{3}(\xi), \\
& g(\xi)=2 \eta_{b}^{1 / 2} E_{1}^{-1 / 2}(\xi, \eta) \cos \frac{1}{3}\{\pi \pm \\
& \left.\cos ^{-1}\left[k E_{1}^{-1 / 2}\left(0, \eta_{b}\right) E_{1}^{1 / 2}\left(\xi, \eta_{b}\right)\right]\right\} \text {, } \\
& E_{1}(\xi, \eta)=\left(\frac{\eta_{b}}{n}\right)^{2} \eta+\frac{1-\sigma}{\sigma}\left(\frac{\eta_{b}}{n}\right)^{2}\left[B_{11}(\xi, a) N_{1}(\xi, \eta, a)\right. \\
& \left.+B_{11}(\xi,-a) N_{1}(\xi, \eta,-a)\right] \text {, } \\
& B_{11}(\xi, a)=1+\operatorname{coth} a \operatorname{coth} \xi\left(\tanh ^{2} \xi-\frac{1-\sigma}{\sigma} \operatorname{sech}^{2} \xi\right) \text {, } \\
& N_{1}(\xi, \eta, a)=\frac{2}{\tanh a+\tanh \xi} \operatorname{tg}^{-1}\left(\left(\tanh \frac{\xi+a}{2} \tan \frac{\eta}{2}\right),\right. \\
& k=\psi_{b} / \psi_{s}, \quad \psi_{s}=\eta_{b}^{3 / 2} E_{1}^{-1 / 2}\left(0, \eta_{b}\right) \text {, } \\
& \cosh a=\sigma \cosh \lambda \text {. }
\end{aligned}
$$


When contraction ratio $n=2$ and the throat wall radius $r=2$, the relation between the throat flow field and the discharge coefficient $k$ is worked out according to equations (19) and (20). Figure 5 gives the iso-Mach number line distribution in the throat with the values of $k=0.5,0.9,0.98$ and 1.0 etc. respectively. For $k=1.0$ sonic line $\mathrm{AE}$, limiting characteristic line $\mathrm{BE}$, dividing line $\mathrm{DE}$ and influence line $\mathrm{CF}$ are also given. From figures $5(\mathrm{a})$ and $5(\mathrm{~b})$, one can see that when $k=0.5$, the flow field as a whole can be approximately considered as incompressible, or low subsonic. When $k=0.9$, the influence of compressibility has to be considered, but the nozzle flow field as a whole is still subsonic; when $k=0.98$, local supersonic regions occur in the vicinity of either side of the throat wall; when $k=1 \cdot 0$, a breakoff occurs in the local supersonic region mentioned above and the sonic lines on the two sides extend until they meet on $x$-axis at the downstream of the throat. The throat flow is thus divided into two parts: the upstream subsonic flow and the downstream supersonic flow. This is the state of flow generally known as the supersonic nozzle flow.

For $\gamma=1.5,1.33 \ldots$ equations (86) similar to equation (20) can be obtained; for any value of $\gamma$, a corresponding result can be obtained by using the integral relation equations (16)-(19). Figure 6 gives the relation between the Mach number at the throat wall and the discharge coefficient $k$ with $\gamma=1.40$ and the throat wall radius, 1,2 and $\infty$. From the figure, we can see that for any given throat wall radius, the throat wall Mach number steadily increases with $k$. The larger the value of $k$, the greater is the rate of the increase.

\subsection{Nozzle flow field}

In the study of transonic flow, the hyperbolic nozzle, being a typical example, is of great significance both in theory and in practice. It has been studied by many scholars
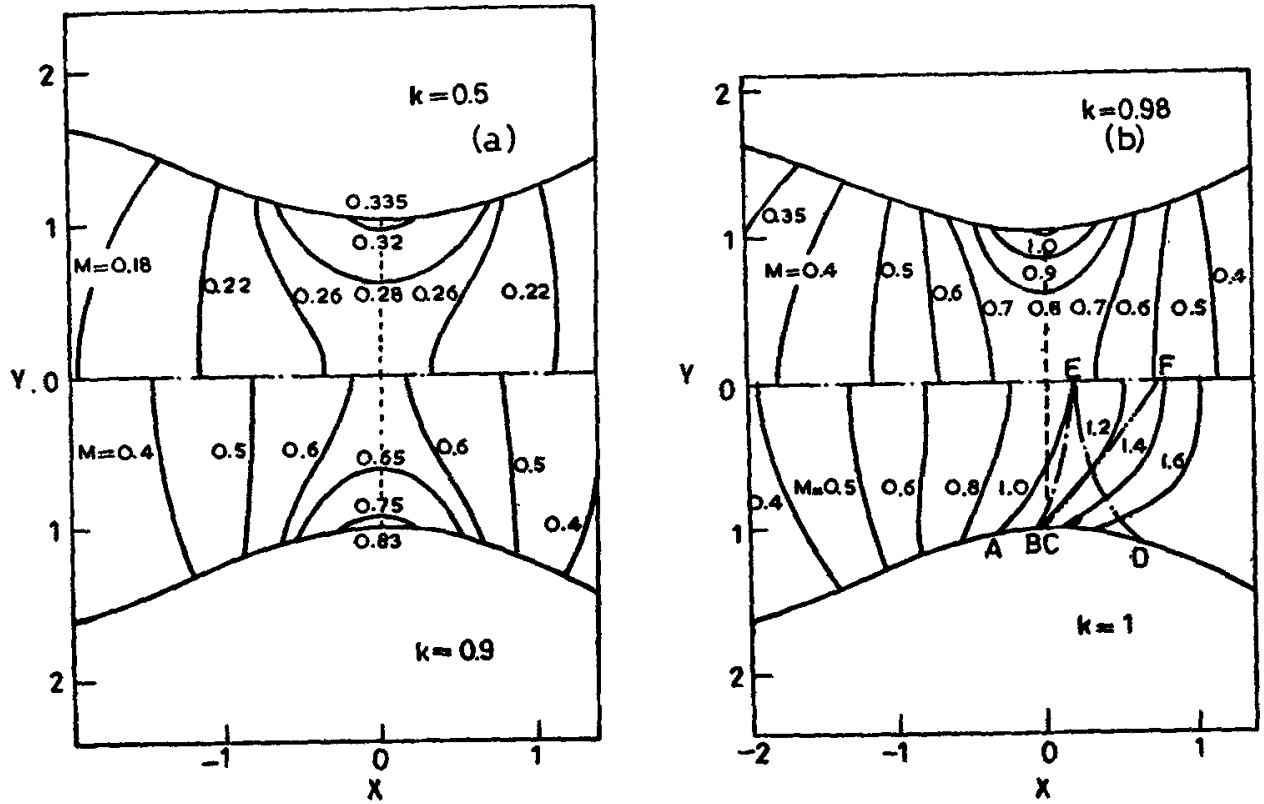

Figure 5. (a) Relation between the Mach number distribution and the discharge coefficient $k$ (for $k=0.5$ and 0.9 ). (b) Relation between the Mach number distribution and the discharge coefficient $k$ (for $k=0.98$ and $1 \cdot 0$ ). 


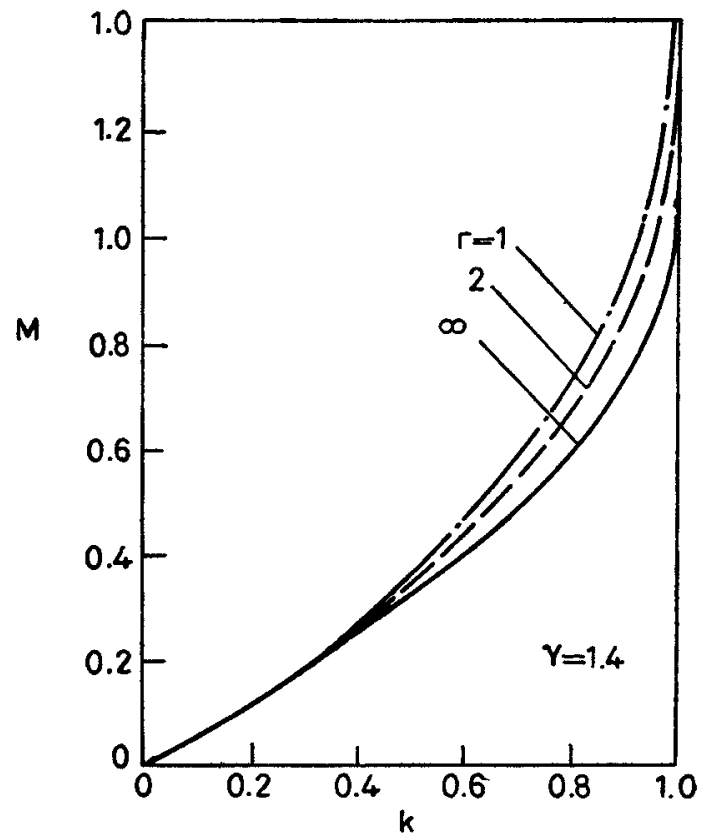

Figure 6. Relation between the maximum Mach number at the nozzle throat and the discharge coefficient $k$.

using various methods: Emmons (1946) used the relaxation method, Cherry (1959) the hodograph method (obtaining an accurate solution), Hall (1962) the small parameter method, and Seria (1972) the time-step correlation method. Most of these studies, however, only concentrated on one or two specific examples. Hall (1962) did discuss general conditions, but his method can only be applied to a nozzle with a relatively large wall radius of curvature. In what follows, we will apply the present method to the determination of the transonic flow (Jiazhen Hue and Lin Tong Ji 1979 ) in the throat region, taking the hyperbolic nozzle as an example.

By using the function of a complex variable, the hyperbolic nozzle coordinate relation between $z$ and $\zeta$, and the inverse of Lamé's coefficients $H$ for the coordinate transformation, can be represented as

$$
\left.\begin{array}{l}
z=\operatorname{cosec} \eta_{b} \sinh \zeta, z=x+i y, \zeta=\xi+i \eta, \\
H(\xi, \eta)=\operatorname{cosec} \eta_{b}\left(\sinh ^{2} \xi+\cos ^{2} \eta\right)^{1 / 2},
\end{array}\right\}
$$

in which the constant $\operatorname{cosec} \eta_{b}$ involves the half-height of the throat. In this coordinate system, when the ratio of specific heats $\gamma$ equals $1+1 / \nu$ of and $\nu$ is a positive integer, the flow field at the throat has a simple analytical solution. For any value of $\gamma$, the computations can be carried out on the basis of formulae (16)-(19). Part of the typical results are given below.

Figure 7 gives the wall contours and the corresponding throat sonic lines for $\gamma=1.40$ and wall radius $r=10.6,5,1,0.22$ respectively. The figure shows that for a comparatively large value of $r$, the sonic line has only one turning point located at its centre; when $r$ is less than $1 \cdot 1$, an additional turning point occurs in the 
vicinity of both sides of the wall surface. Within the range given in the figure, the distance between the centres of the sonic line and the throat section increases monotonically as $r$ decreases, but with the continuous decrease in the value of $r$ below $0 \cdot 18$, the distance begins to decrease.

Figure 8 gives the Mach number distribution on the central streamline for $\gamma=1.4$ and $r=10 \cdot 6,5,1 \cdot 0$, and $0 \cdot 22$ respectively. It is evident from the figure that all Mach number distribution curves intersect at one point, whose location is given approximately as $x=0.37$ and $M=1.08$. Reduction in the value of $r$ is accompanied by rapid variation in the airflow acceleration.

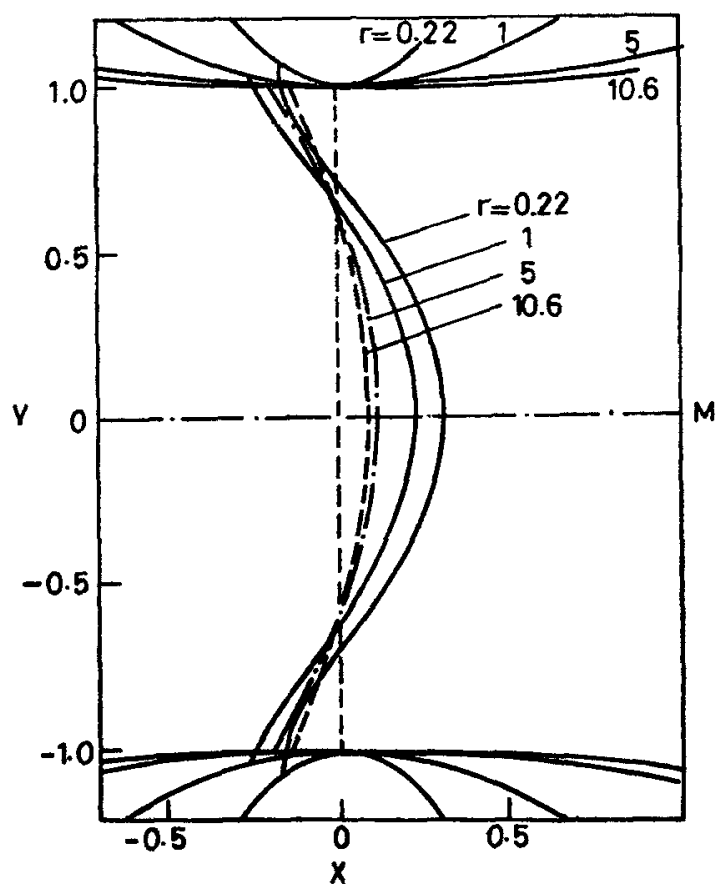

Figure 7. Sonic lines for hyperbolic nozzles.

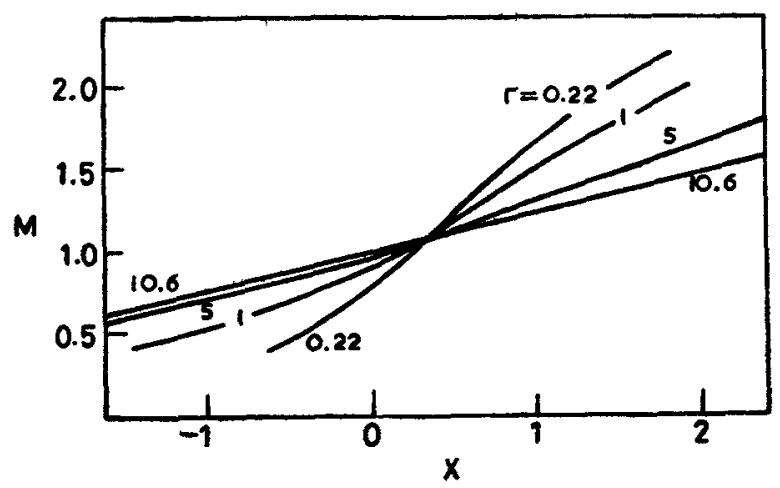

Figure 8. Mach number distribution along the axis of hyperbolic Laval nozzles. 
Figure 9 demonstrates the influence of $\gamma$ upon the iso-Mach number distribution in the throat flow. The dotted lines are for $\gamma=1 \cdot 2$ and the solid lines are for $\gamma=1.667$. It is obvious from the figure that the larger the value of $\gamma$, the closer the iso-Mach line is to the throat.

Figure 10 gives the comparison between the results obtained by the present method and those obtained by Cherry (1959) and by Seria (1972) at $\gamma=1.40$ and $r=2$. Cherry, using the hodograph method, obtained the exact solution for the quasihyperbolic contour; Seria using the time step correlation method, carried out the calculations for the hyperbolic nozzle. The figure shows that the results obtained by the present method and those by Seria coincide for the iso-Mach lines when $M=0.46$ and 1.90 . When $M=0.75,1.32$ and 1.68 our results lie between those obtained by Cherry and Seria, and our results for the sonic line coincide with those obtained by Cherry. But compared with both Cherry's and Serra's method, the

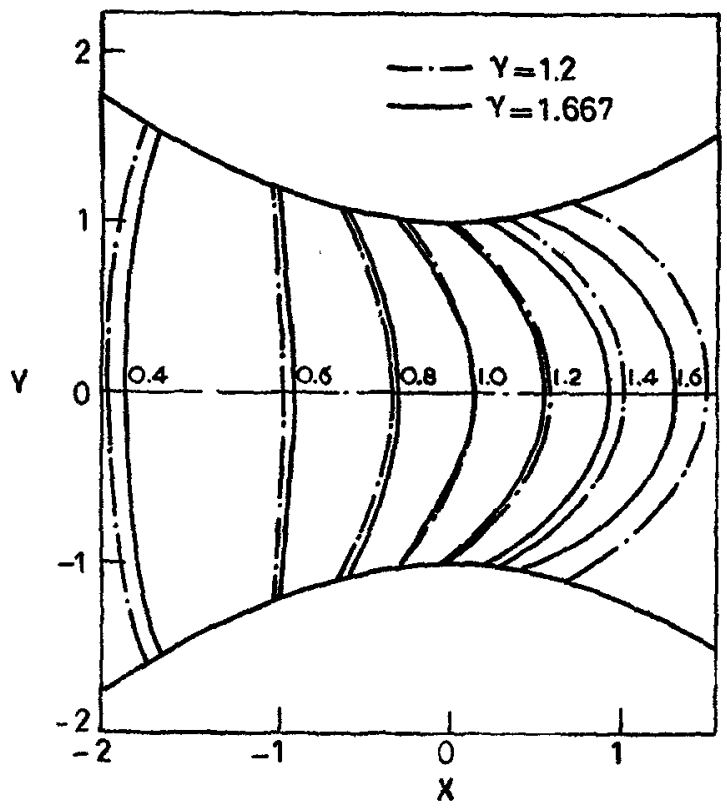

Figure 9. Influence of the ratio of specific heats upon the flow-field.

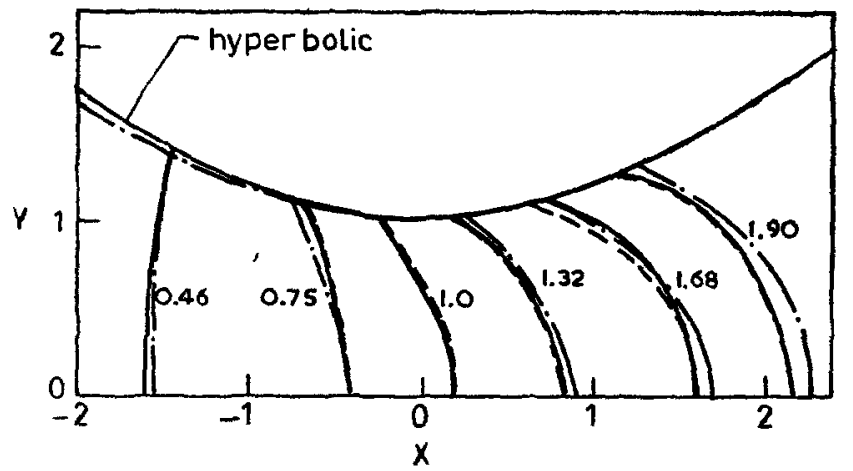

Figure 10. Iso-Mach line distribution for a hyperbolic nozzle. _- Present results; - . Cherry (1950); _ _ - Seria (1972). 
present method is much simpler, and furthermore, the nozzle throat contours can be selected according to need.

\subsection{Axisymmetric nozzle flow field}

The method given in this paper is also applicable to the axisymmetric nozzle throat flow with any wall contour. In view of the extensive use of the circular arc throat, this section intends to analyse briefly the transonic flow for this type of nozzle.

For this purpose, the employment of double circular coordinates (Lamb 1932) will prove convenient. The coordinate relations, and $H\left(\xi, \gamma_{l}\right)$, the inverse of Lamés coefficients for the coordinate transformation, are respectively

$$
\left.\begin{array}{l}
z=\operatorname{coth} \dot{\eta}_{b} \tan \zeta, \quad z=x+i y, \quad \zeta=\xi+i \eta, \\
H(\xi, \eta)=\frac{2 \operatorname{coth} \eta_{b}}{\cosh 2 \eta+\cos 2 \xi} .
\end{array}\right\}
$$

The constant coth $\eta_{b}$ is so chosen that the throat radius is 1 . Using the above-mentioned conformal curvilinear coordinates and applying the equations (16) (19), one obtains the transonic flow ficld solution for the circular arc throat region. For some common ratios of specific heats, e.g. for $\gamma=1 \cdot 1,1 \cdot 667,1 \cdot 2$ etc., the throat flow field solution can be expressed by simple analytical equations (Jiazhen Hue and Lin Tong Ji). For instance when $\gamma=1 \cdot 4$, the resulting formulae are

$$
\begin{aligned}
& \psi(\xi, \eta)=k_{a} g(\xi)[F(\lambda)-F(\mu \lambda)] \\
& F(\lambda)=\frac{2}{15}\left(23-11 \lambda+3 \lambda^{2}\right)(1-\lambda)^{1 / 2}-2 \tanh ^{-1}(1--\lambda)^{1 / 2} \\
& k_{a} g(\xi)\left[F\left(\lambda_{b}\right)-F\left(\mu_{b} \lambda_{b}\right)\right]=\mu_{s} \\
& \psi_{s}=4 \sqrt{6} k_{a} r(1+2 r)^{-1 / 2} \lambda_{s}^{1 / 2}\left[\left(1-\mu_{s} \lambda_{s}\right)^{5 / 2}-\left(1-\lambda_{s}\right)^{5 / 2}\right] \\
& F\left(\lambda_{s}\right)-F\left(\mu_{s} \lambda_{s}\right)+2\left(1-\lambda_{s}\right)^{5 / 2}-2\left(1-\mu_{s} \lambda_{s}\right)^{5 / 2}=0 \\
& \lambda=\lambda(\xi, \eta)=\frac{1}{24}(1+2 r)^{-1}(\cosh 2 \eta+\cos 2 \xi)^{2} g^{2}(\xi) \\
& \lambda_{b}=\lambda\left(\xi, \eta_{b}\right), \lambda_{s}=\lambda\left(0, \eta_{b}\right) \\
& \mu=\mu(\xi, \eta)=\cos ^{4} \xi(\cosh 2 \eta+\cos 2 \xi)^{-2} \\
& \mu_{b}=\mu\left(\xi, \eta_{b}\right), \mu_{s}=\mu\left(0, \eta_{b}\right) \\
& k_{a}=\frac{1}{2}\left(\frac{r+1}{2}\right) \frac{1}{r-1}(1+2 r)^{1 / 2}
\end{aligned}
$$

Using the above method, we obtain the following transonic flow field for the circular arc nozzle for different values of $\gamma$ (Jia Zhen Hue and Lin Tong Ji, to be published).

Figure 11 gives the relationship between the maximum nozzle discharge $\psi_{s}$ and the 
wall radius $r$, for $\gamma=1.40$. At the same time, results of some other theoretical and experimental studies are compared. It is evident from the figure that when $r$ is comparatively large, the results obtained by the present method and those by Hall (1962) are basically the same, while the results given by Sauer (1944) are somewhat small; Kliegel \& Levine's results (1969), on the contrary are somewhat large since they used the inverse of $(1+r)$ as a small parameter, thus giving rise to a slowly convergent series. With the decrease in the value of $r$, Hall's solution becomes divergent, and Sauer's is no longer valid. The results given by Kliegel \& Levine are evidently too large. Klopfer \& Holt (1975), utilizing the integral relation method, obtained better results than those of Kliegel \& Levine. The diagram shows that our results lie between those obtained by Klopfer \& Holt on the one hand and Sauer on the other. Moreover, they match better with the experimental results (Back et al 1965; Durham 1955; Norton \& Sheiton 1969; Back \& Cuffel 1971). At the same time, one must notice that when the entry portion is non-circular, e.g. when it is conical in shape, $\psi_{s}$ is increased, especially when $r$ is comparatively small.

Figure 12 gives the relation between the wall radius and the Mach numbers on the wall surface and on the axis at the throat section; at the same time, a comparison is made with the results of Sauer (1944), Hall (1962) and Kliegel \& Levine (1969). It can be seen from the diagram that for relatively large values of $r$, the results given by the present method and those by the others are basically identical; when $r$ is relatively small, the Mach numbers at the wall given by Kliegel \& Levine are relatively high while those at the axis are relatively low. These results coincide with those presented in figure 7.

Figure 13 gives the contours and location of the sonic lines for $\gamma=1.40$ and wall radius of curvature $r=10,5,1$ and 0.50 respectively. The figure indicates that for relatively large $r$ values, the sonic lines are nearly vertical; with the gradual reduction of the $r$ value the curvature of the sonic line increases. When $r$ is smaller than 0.5 , there appears a second turning point in the vicinity of each side of the wall surface, in addition to the original one at the centre of the sonic line.

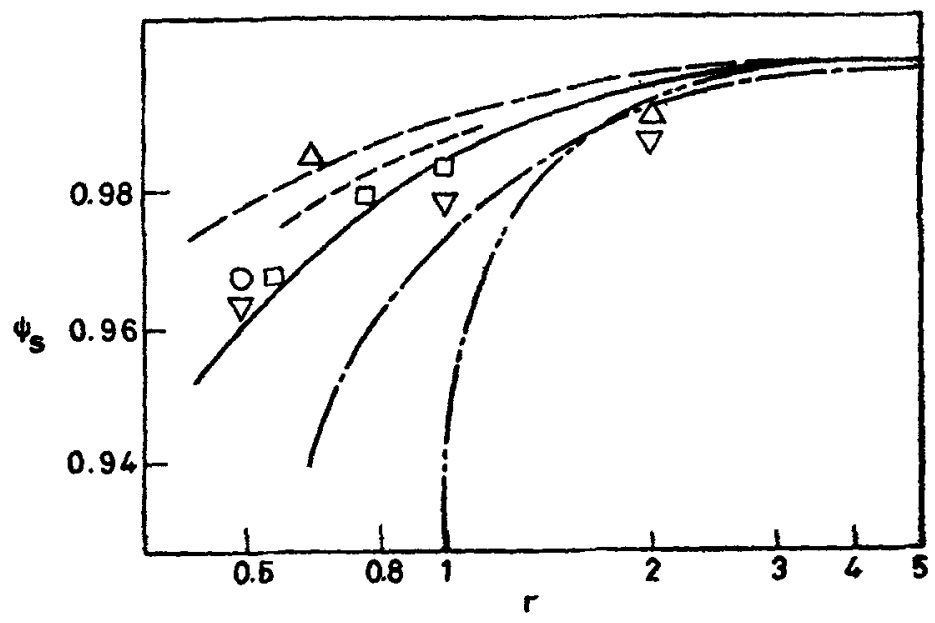

Figure 11. Maximum discharge for axisymmetric Laval nozzles. _- Present method; - - Sauer (1944); -..-Hall (1962); - Kleigel \& Levine (1969); Klopfer \& Holt (1975); $\triangle$ Back et al (1965); $\nabla$ Durham (1955); $\square$ Norton \& Shelton (1969); O Back \& Cuffel (1971). 


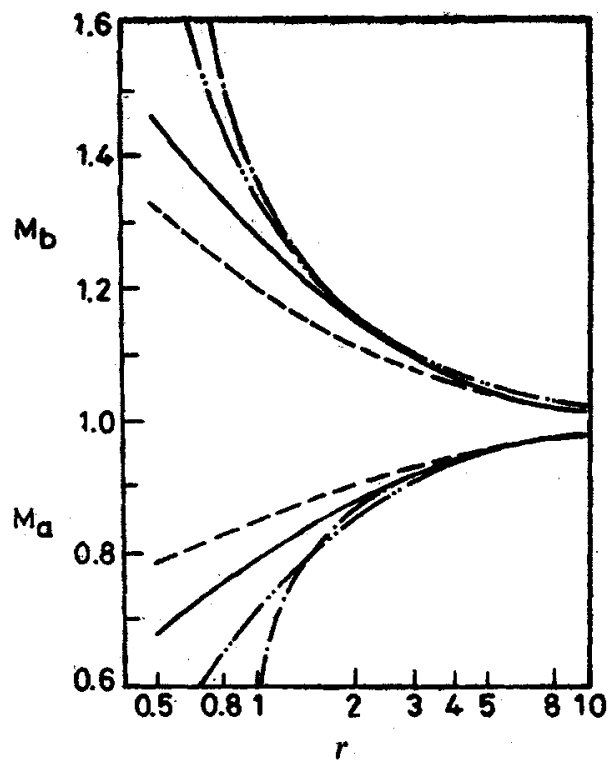

Figure 12. Throat wall and centre line Mach numbers for axisymmetric Laval nozzles. _- Present results; _- Sauer (1944); _-... Hall (1962); Kliegel \& Levine (1969).

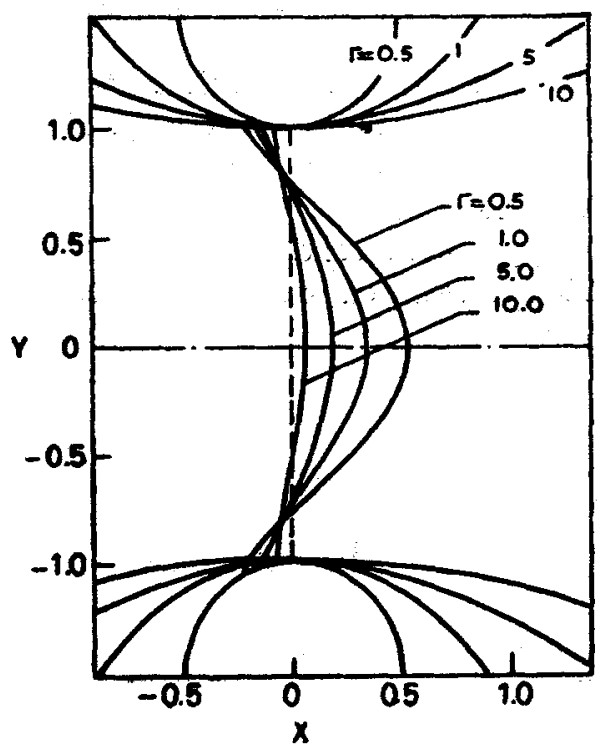

Figure 13. Sonic lines for axisymmetric Laval nozzles of the circular arc type.

Figure 14 gives the typical iso-Mach number distribution of the transonic flow in the throat for $r=1$

Figure 15 shows the influence of $y$ upon the maximum discharge $\psi_{s}$. It is clear that with the increase of the $\gamma$ value the corresponding $\psi_{z}$ value decreases.

It is to be noted that the present method, besides being simple in computation, is applicable to a small throat wall radius as well as to a large one. 


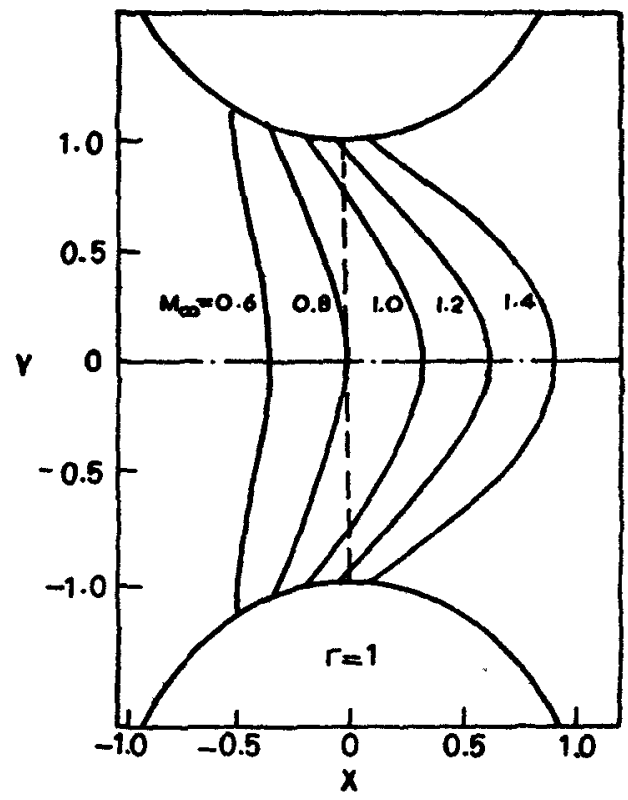

Figure 14. Transonic flow field for an axisymmetric Laval nozzle of the circular arc type.

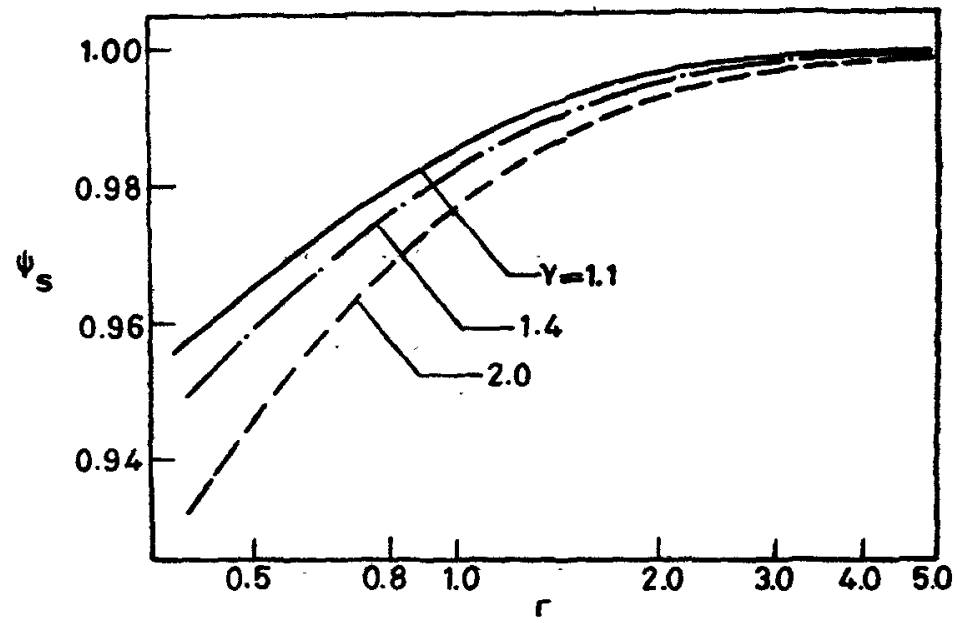

Figure 15. Effect of specific heat ratio on the maximum discharge of axisymmetric Laval nozzles.

\section{External flow past bodies}

In this section, the external flow past a body is studied in the light of the present method. A method of choosing the far field computation boundary is proposed; to overcome the difficulties associated with the small disturbance theory in dealing with thick bodies. As examples of the method, flow around a circular cylinder and a sphere aro presented with free-stream Mach number $M_{\infty}$ varying from subcritical to supercritical including $M_{\infty}$ equal to 1 . 


\subsection{Computation boundary}

In tackling the problem of flow past a body, any numerical method, in general, can only provide solutions in a limited space (region). Therefore, how to select a distant computation boundary is a problem of great significance. In the case of a thin wing or a slender body, the disturbance generated in the flow field is usually small; so at a finite distance from the body the conditions at infinity can be applied. For example, for the flow field around a thin wing, Emmons (1948) Murman \& Cole (1971) and others have replaced the boundary at infinity by one at a distance of a few chord lengths from the body. Chan et al $(1974,1975)$ divided the flow surrounding the body into the near region and the far region. A relatively fine mesh is used for the near region and a relatively coarse one for the far region. The two regions are matched at their junction. Disturbances to the flow field by thick bodies are usually large, especially under transonic conditions. It does not matter whether a straight line or a regional envelope line is selected as the outer computation boundary; they both need to be located at a very great distance from the body; this has brought about great difficulties in computation, as the region involved is greatly enlarged.

It is expected that the greater the distance from the body, the smaller is the difference between the streamlines of a compressible flow and the corresponding streamlines of an incompressible flow. Therefore, it would be a better approximation to select the streamlines of an incompressible flow at a certain distance from the body as the computation boundary. With the use of the conformal curvilinear coordinates in this paper, such a boundary then is the coordinate line $\eta=\eta_{b}$, which greatly facilitates the computations involved.

But it is to be noted that for flow around a body, there is a section between the computation boundary $\eta_{b}$ and the body surface $\eta=0$ which is the narrowest. Take the circular cylinder as an example; such a section occurs at $\xi=0$, i.e. on the $y$ axis. The mathematical expression for this relation can be written as

$$
y(0, \eta)-y(0,0)<y(\xi, \eta)-y(\xi, 0) .
$$

Moreover, for the oncoming uniform flow at an infinite distance, i.e. $\xi=-\infty$, the discharge $\psi_{b}$ between $\eta=0$ and $\eta=\eta_{b}$ is $(\rho u)_{\infty} \eta_{b}$. Therefore, the passage of the discharge $\psi_{b}$ through the narrowest section on $\xi=0$ serves as the necessary condition for $\eta_{b}$ to be a possible computation boundary for the transonic flow. Using (7) and the condition $\xi=0$, this relation can be written as (Lin Tong Ji \& Jia Zhen Hue 1980)

$$
\begin{gathered}
\left(\frac{(\gamma+1)}{2}\right)^{\frac{1}{\gamma-1}} g(0) \int_{0}^{\eta_{b}}\left[1-\frac{\gamma-1}{\gamma+1} \frac{g^{2}(0)}{H_{i}^{2}(0, \eta)}\right]^{\frac{1}{\gamma-1}} \\
\times \frac{H_{2}(0, \eta)}{H_{1}(0, \eta)} y^{\prime}(0, \eta) d \eta=(\rho u)_{\infty} \eta_{b} .
\end{gathered}
$$

For (24) to be valid, there must exist a minimum value of $\eta_{b}$ for a given supercritical free-stream condition $(\rho u)_{\infty}$ or $M_{\infty}$. Such a value is defined as the characteristic 
boundary and is represented by $\eta_{c^{*}}$. From (24), one can see that $\eta_{c}$ is only a function of $g(0)$; as a result of the above definition, $\eta_{c}$ will satisfy the extremal relation

$$
\partial \eta_{b} / \partial g(0)=0
$$

With due note taken of the fact that the upper limit in the integral is a variable, and replacing the $\eta_{b}$ in (24) by $\eta_{c}$ and solving the equation, we obtain the following equation for $\eta_{c}$ to satisfy the extremal relation mentioned above:

$$
\begin{gathered}
\int_{0}^{\eta_{c}}\left[\frac{g^{2}(0)}{H_{1}^{2}(0, \eta)}-1\right]\left[1-\frac{\gamma-1}{\gamma+1} \frac{g^{2}(0)}{H_{1}^{2}(0, \eta)}\right]^{\frac{2-\gamma}{\gamma-1}} \\
\times \frac{H_{2}(0, \eta)}{H_{1}(0, \eta)} y^{\epsilon}(0, \eta) d \eta=0 .
\end{gathered}
$$

Now, the characteristic boundary $\eta_{c}$ for a given oncoming flow Mach number can be obtained by replacing $\eta_{b}$ in (24) by $\eta_{c}$ and linking it with (25). When the oncoming flow Mach number $M_{\infty} \rightarrow 0$, the solution of the simplified equation (12) is accurate for the whole space; therefore, the corresponding computation boundary $\eta_{b}$ should be $\infty$. When $M_{\infty}=1,(24)$ and (25) yield $\eta_{c}$ as $\infty$. Since $\eta_{b}>\eta_{c}, \eta_{b}$ is also $\infty$. This shows that for $M_{\infty}=1$, one gets the maximum value of the oncoming flow discharge and the effect of the body upon the flow field is to be shared by the whole flow field. This is precisely what is charactersitic of a transonic flow. When the oncoming stream reaches the critical Mach number, the body-induced effect upon the flow field is mostly borne by the near region; $\eta_{c}$ is then comparatively small in value.

To sum up, as $M_{\infty}$ gradually increases from 0 to the critical Mach number and then to 1 , the corresponding values of $\eta_{b}$ first gradually decrease from $\infty$ to a minimum value and then gradually increase to $\infty$. As a result, one can write

$$
\eta_{b}=\eta_{c}+\sum_{k=1}^{n} a_{k} M_{\infty}^{-b^{k}}, 0 \leqslant M_{\infty} \leqslant 1
$$

A proper selection of $\eta_{b}$ will raise the accuracy of the first order solution and reduce the number of iterations. For example, for the flow past a circular cylinder, with $n=a_{1}=b_{1}=1$, (26) yields very good results. A detailed discussion of the topic will be given below.

\subsection{Flow past a circular cylinder}

The transonic flow past a circular cylinder is a longstanding problem that has engaged the attention of many researchers. For instance, Imai (1941) used the frce stream Mach number as a small parameter to calculate the flow around a circular cylinder with $M_{\infty}<0.4$; Cherry $(1947,1949 \mathrm{a}, \mathrm{b})$ used the hodograph method, to simulate, by repeatcd additions of basic potentials, the approximate flow contours of a quasicircular cylinder and to give a special solution for $M_{\infty}=0.51$. Simanski (1956) 
used a method similar to that of Imai (1941), and chose higher-order terms for calculations about subcritical flow past a circular cylinder. Dorodynitsyn (1956) and Chushkin (1957) used the strip integral method to calculate the flow past a circular cylinder for $M_{\infty}=1$. Later, Holt \& Masson (1970) used the integral relation method to calculate the critical flow past a circular cylinder. Moretti (1970) used the time-step correlation method for $M_{\infty}=1 \cdot 0$, and Norrie \& Vries (1975) used the finite element method for $M_{\infty}=0.3$ and 0.7 . Because of the inherent limitations of these methods, they could only provide results about the flow under certain specific conditions. The present paper, on the other hand, by using the method of conformal curvilinear coordinates, manages to provide an overall picture for the flow past a circular cylinder and gives systematic results ranging from subcritical to supercritical, including freestream Mach number $M_{\infty}=1$.

Let us assume that the free stream at infinity and the $x$-axis are parallel and that the origin of the coordinate system is situated at the centroid of the cross section. Then, for a unit circle in the $x y$ plane, the relations between the equipotential lines and the streamlines $(\xi, \eta)$ for an incompressible potential flow and the coordinates $(x, y)$, and the related Lamé cosfficient, are given respectively as (Lamb 1932; Lin Tong Ji \& Jia Zhen Hue 1980)

$$
\left.\begin{array}{l}
\zeta=z+\frac{1}{z}, z=x+i y, \zeta=\xi+i \eta, \\
h=\frac{1}{H}=\frac{1}{x^{2}+y^{2}}\left[\left(x^{2}+y^{2}\right)^{2}-2 x^{2}+2 y^{2}+1\right]^{1 / 2} .
\end{array}\right\}
$$

Substituting (27) into (24) and (25), (12) yields the first-order solution. Taking the streamlines thus obtaincd and their orthogonal lines as curvilinear coordinates, a second-ordcr solution can be worked out by the same method, and so on to higherorder approximations. Generally speaking, the selection of the computation boundary $\eta_{b}$ is not critical, but an appropriate selection can enhance the accuracy of the first-order solution and reduce the number of iterations necessary in the numerical operation. The results of computations based upon (26) show that for flow past a circular cylinder, with $n=a_{1}=b_{1}=1$, i.e. $\eta_{b}=\eta_{c}+1 / M_{\infty}$, even the first-order approximation has a very good accuracy, as is shown in figures 17,18 and 19.

The curves relating $g(\xi)$ to the free-stream Mach number $M_{\propto}$ for different values of $\xi$ are shown in figure 16 . It can be seen that when $\xi \rightarrow-\infty$ the $g$ value equals the corresponding free-stream velocity, i.e. $g(-\infty)=u_{\infty}$. This indicates that at infinite distance there is no body-induced disturbance and that the compressible and the incompressible flow are identical; with the increase in $\xi$, the influcnce of compressibility also grows. Departure from the free stream begins with $g<u_{\infty}$; this indicates that the influence of compressibility has reduced the flow velocity and made it lower than that in a corresponding incompressible fiow. On approaching the forward stagnation point, which for a circular cylinder is at $\xi=-2$, the value of $g$ is the smallest. With the continued increase in $\xi$, the value of $g$ rises until it equals $u_{\infty}$ at the location where $\xi=\xi_{m}$. It has to be noted, however, that the location of $\xi_{m}$ varies with $M_{\infty}$. For still larger values of $\xi$, the values of $g$ approach $u_{\infty}$. At $\xi=0$, i.e. at the thickest section of the circular cylinder, the value of $g$, i.e. the influence of compressibility, is the largest. 


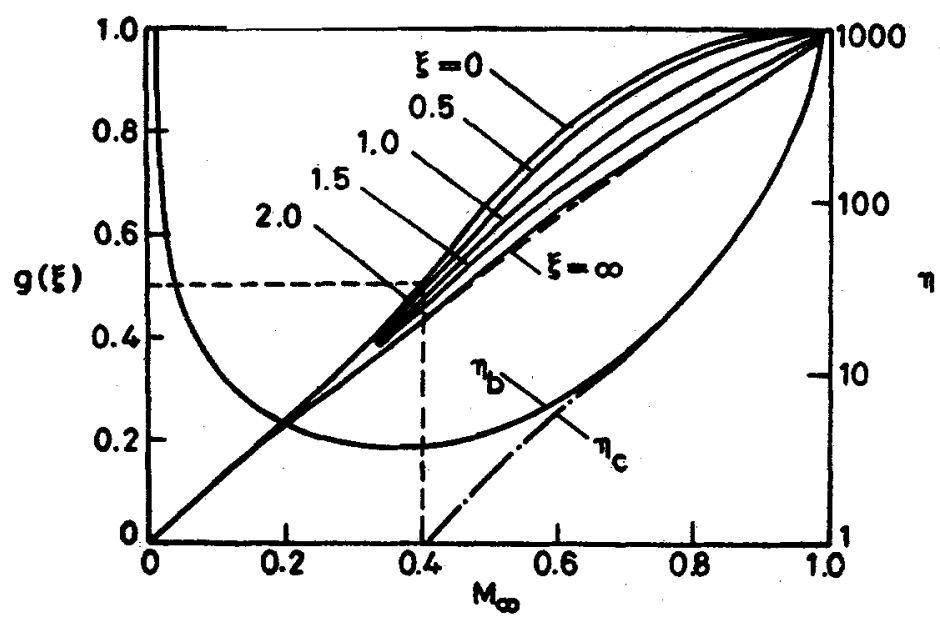

Figure 16. "Discharge functions" for a circular cylinder.

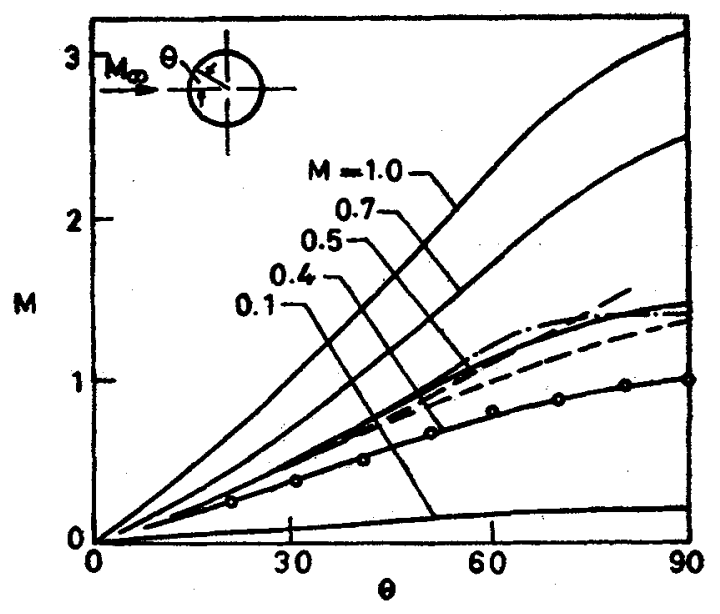

Figure 17. Mach-number distribution on the surface of a circular cylinder. Present results; _ _ _ Dorodnitsyn (1956); _- - - Cherry (1949); O O Imai (1941); Hafez et al (1979).

From the results obtained in this paper, one can see that the values of $g$ represent the effect of compressibility Whether the body is thick or thin, the variation of $g(\xi)$ with $\xi$ tends to follow a similar pattern. But, the difference between the maximum and the minimum value of $g$ for a thin body is very small; therefore, one can go a step further towards approximation by using a single compressibility correction value for the entire flow field. For example, the Karman-Tsien method(Tsien 1939; Von Karman 1941) has yielded very good results for thin wings. As for thick bodies, from the results obtained by the present method, one can see that for different $\xi$ values, it is necessary to use different compressibility corrections, namely compressibility corrections should be a function of the parameter $\xi$. Moreover, for different values of free stream Mach number $M_{\infty}$, the compressibility corrections are also different. Figure 16 shows that for very low $M_{\infty}$, the effect of compressibility is very small; with the increase in $M_{\infty}$, the compressibility correction also increases; at the critical Mach 


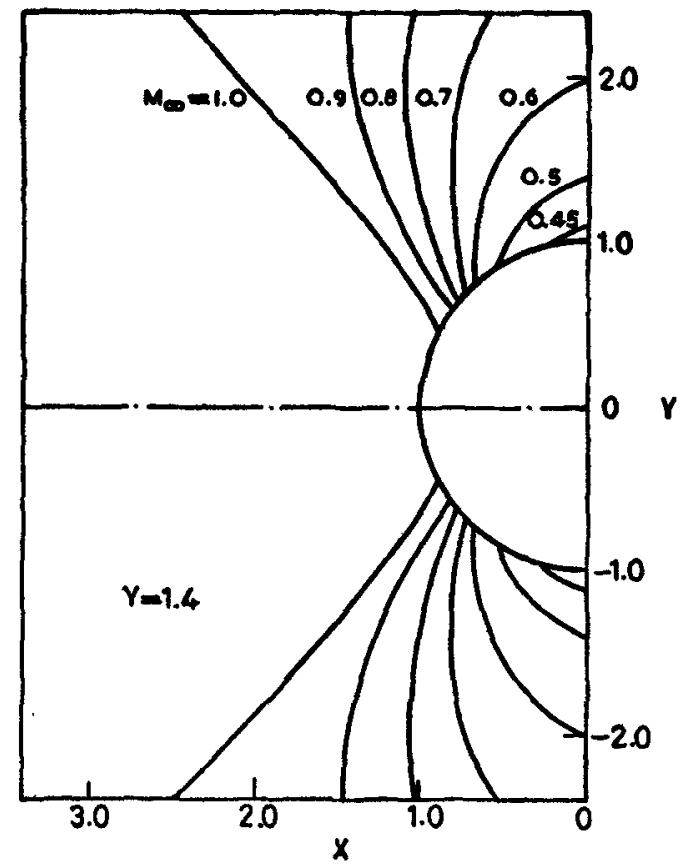

Figure 18. Sonic lines for the flow past a circular cylinder.

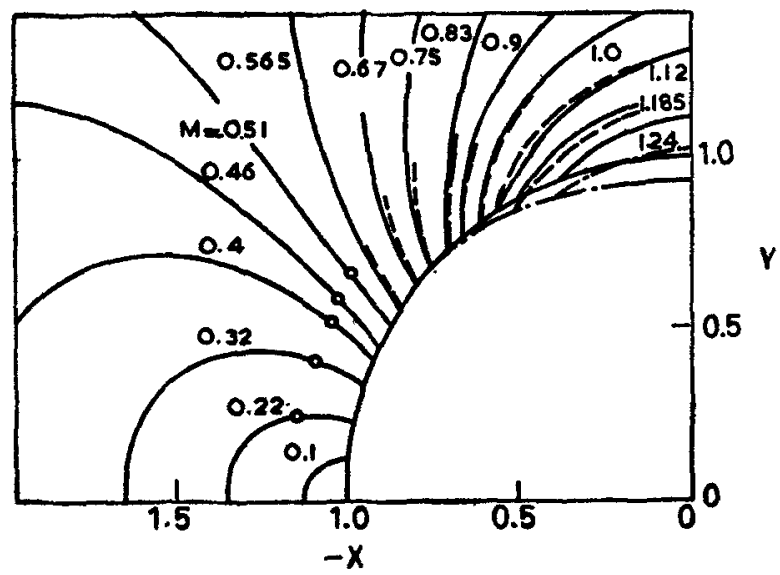

Figure 19. Iso-Mach lines for the flow past a circular cylinder at $M_{\infty}=0.51$. - Present results; - - Cherry (1949).

number, the correction reaches a maximum; further increase in $M_{\infty}$ will cause, instead, a decrease in the values.

Figure 17 gives the Mach number distribution on a circular cylinder surface with frce-stream Mach numbers ranging from 0.1 right up to 1 . For the sake of comparison, the figure also gives Cherry's result (1949b) at $M_{\infty}=0.51$; Chushkin's result (1957) at $M_{\infty}=1$ and Imai's result (1941) at $M_{\infty}=0.4$.

Figure 18 gives the sonic lines for flow past a circular cylinder with $M_{\infty}$ varying from 0.45 up to 1 .

Figure 19 gives the flow field past a circular cylinder at $M_{\infty}=0.51$ and $\gamma=1.405$. For comparison, the figure also gives Cherry's (1949b) quasi-circular cylinder results 
(dotted lines). From the figure it can be seen that for $\theta \leqslant 45^{\circ}$, Cherry's quasi-circular cylinder contours coincide with those of a real circle and that his iso-Mach lines coincide with ours. When $\theta>45^{\circ}$, the deviation between the quasi-circular and the real circular cylinder gradually increases. At $\theta=90^{\circ}$, the corresponding deviation reaches a maximum of $7 \%$; and the corresponding iso-Mach lines also gradually diverge, as may be expected.

\subsection{Transonic flow past a sphere}

Let us assume that the origin of the rectangular coordinates $(x, y)$ is at the centre of the sphere with the $x$-axis parallel to the free stream at infinity. Because of axisymmetry, one need only consider the flow in the meridional plane $(x, y)$. For a unit sphere, the incompressible potential $(\xi, \eta)$ in the meridional plane $(x, y)$ is (Lamb 1932)

$$
\xi=x\left[1+\frac{1}{2\left(x^{2}+y^{2}\right)^{3 / 2}}\right], \quad \eta=\frac{y^{2}}{2}\left[1-\frac{1}{\left(x^{2}+y^{2}\right)^{3 / 2}}\right] .
$$

From (2) and (28) one obtains the inverse of Lamés coefficients $H_{1}$ and $H_{2}$ as

$$
\begin{aligned}
& \left.H_{1}=\left(x^{2}+y^{2}\right)^{5 / 2}\left\{\left[\left(x^{2}+y^{2}\right)^{5 / 2}-x^{2}+\frac{1}{2} y^{2}\right]^{2}+\frac{9}{4} x^{2} y^{2}\right\}^{-1 / 2},\right\} \\
& H_{2}=\frac{H_{1}}{y}
\end{aligned}
$$

Now, by substituting (28) and (29) into (24) and (25), we obtain the corresponding characteristic boundary $\eta_{c}$ for different values of $M_{\infty}$. As a first order approximation, we select the computation boundary to be equal to $\eta_{c}+\left(M_{\infty}\right)^{-1 / 2}$. According to (28), (29), (4), (12) and (13), one obtains the values of $g(\xi)$ and the flow fields for the corresponding free stream (see figures 20 and 21).

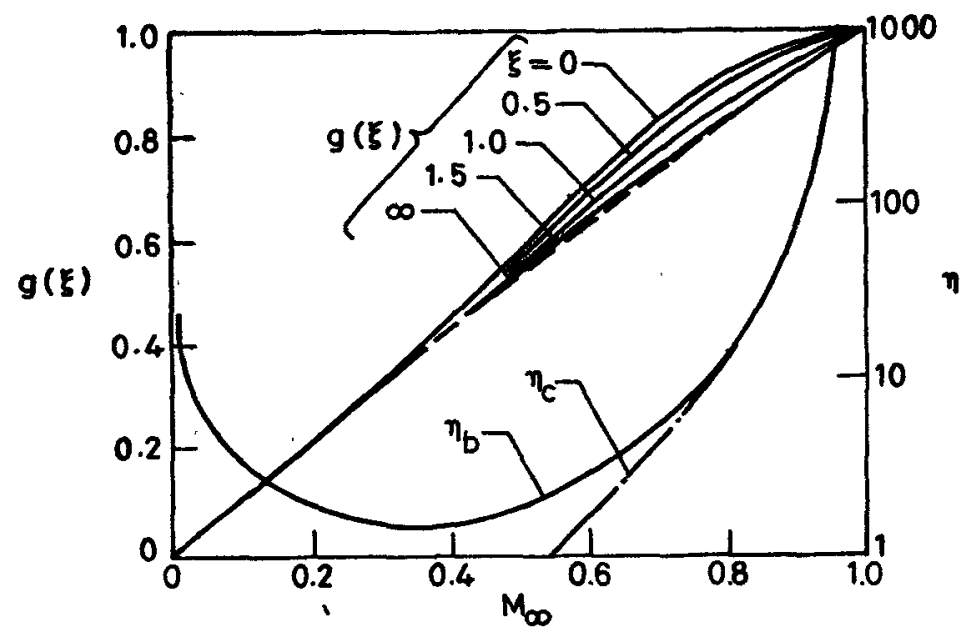

Figure 20. "Discharge function" for a sphere. 


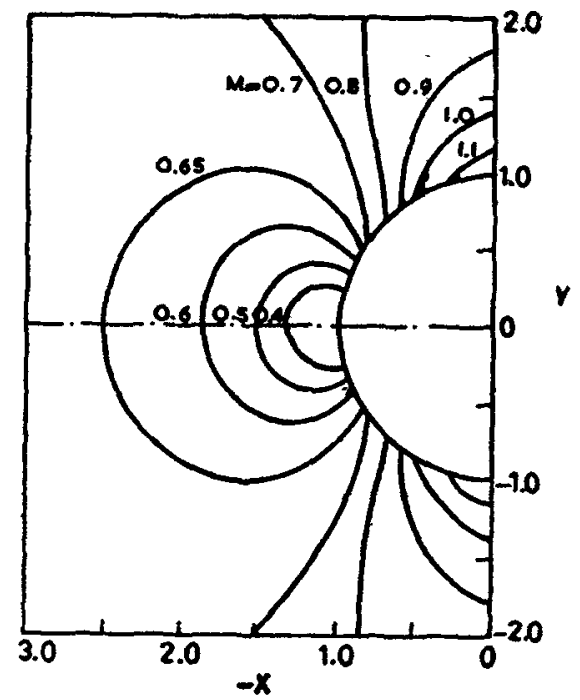

Figure 21. Iso-Mach lines for flow past a sphere at $M_{\infty}=0.7$.

After comparing figure 16 with figure 20, one can see that the critical Mach number for a circular cylinder is 0.403 , whereas for a sphere it is 0.58 . As an example, figure 21 gives the transonic flow about a sphere at $M_{\infty}=0.7$. It shows that the maximum Mach number in the flow field is 1.32 whereas figure 17 indicates that for a circular cylinder the maximum Mach number is $\mathbf{2 \cdot 4 2}$. Moreover, the supersonic region for flow about a cylinder is much larger than that about a sphere.

\subsection{Flow field at $M_{\infty}=1$.}

This is a crucial problem in transonic studies. As a result of the mixed nature of the flow equation, the scope of the body-induccd flow disturbance is especially large, and this causes great difficulties in theoretical analysis, numerical computations and experimental studies. In the case cf a circular cylinder, for example, at $M_{\infty}=1 \cdot 0$, neither the sonic line contours nor the iso-Mach line distribution, nor the characteristics of the far field flow, have yet been made clear. This paper, therefore, intends to discuss, in the light of the method it proposes, the characteristics of the flow at $M_{\infty}=1.0$ for both a circular cylinder and a sphere.

According to the equations given earlier, with $M_{\infty}=1,(24)$ and (25) yield $\eta_{c}=\infty$, therefore $\eta_{b}$ is also $\infty$. Substituting the boundary conditions in the general solution for the stream-function (12), one gets $g(\xi) \equiv 1$. Using (4) and (13) as well as (27) and (28), and assuming $x=r \cos \theta$ and $y=r \sin \theta$, one obtains the Mach number at $(r, \theta)$ as follows:

$$
\left.\begin{array}{rl}
M^{2} & =\frac{r^{4}-2 r^{2} \cos 2 \theta+1}{r^{4}-\frac{\gamma-1}{2}\left(1-2 r^{2} \cos 2 \theta\right)} \text { (circular cylinder), } \\
& \left.=\frac{\left(1+2 r^{3}\right)^{2}+3\left(1-4 r^{3}\right) \cos ^{2} \theta}{4 r^{6}-\frac{\gamma-1}{2}\left[1+4 r^{3}+3\left(1-4 r^{3}\right) \cos ^{2} \theta\right]} \text { (sphere) }\right)
\end{array}\right\}
$$


The flow fields calculated from (30) are given in figures 22 and 23 respectively. It can be seen that for $M>1$, the iso-Mach lines intersect the $y$-axis, whereas for $M<1$ they intersect the $x$-axis. The sonic lines extend to infinity. For a circular cylinder they are represented by that part of the hyperbolic curve which lies outside the cylinder, i.e., by the equation:

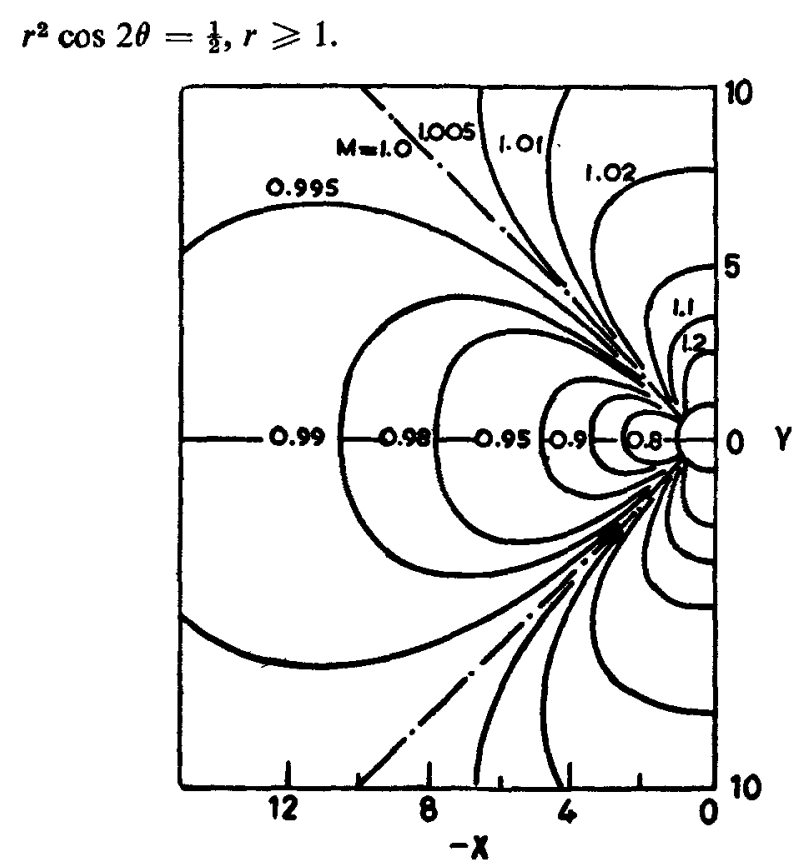

Figure 22. Iso-Mach lines for flow past a circular cylinder at $M_{\infty}=1$.

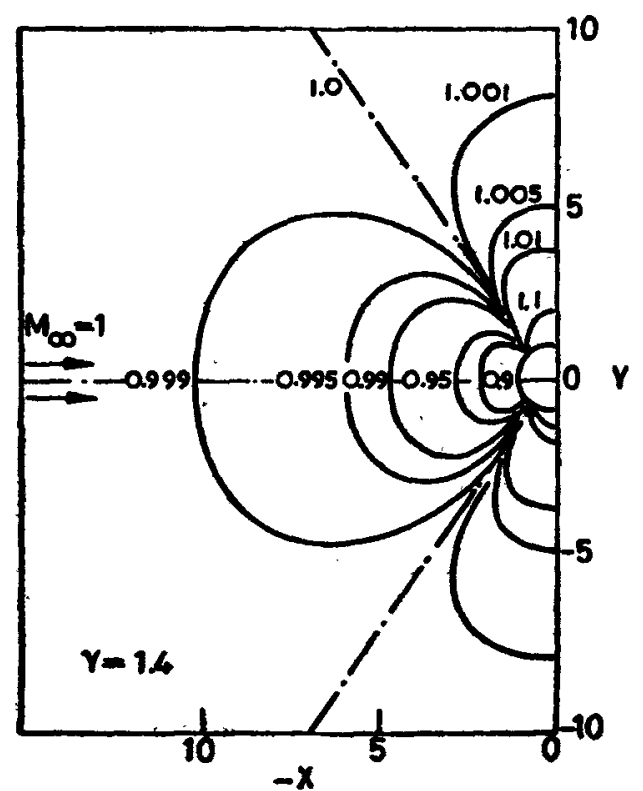

Figure 23. Iso-Mach lines for flow past a sphere at $M_{\infty}=1$. 
These sonic lines begin at $\theta= \pm 30^{\circ}$ on the surface of the cylinder and approach the lines $\theta= \pm 45^{\circ}$ asymptotically. In the case of a sphere, the sonic lines are given by the formula

$$
\cos ^{2} \theta=\frac{4 r^{3}+1}{3\left(4 r^{3}-1\right)}
$$

They begin at $\theta= \pm 41^{\circ} 49^{\prime}$ on the surface of the sphere and approach the conical surface $\theta= \pm 54^{\circ} 45^{\prime}$ asymptotically.

From (30) or (22) and (23), one obtains the deviation of the Mach number on the $x$ and $y$ axis as

$$
\triangle M=M-M_{\infty}=\mathrm{O}\left(r^{-(2+\epsilon)}\right)
$$

Thus the velocity disturbance in this transnoic flow field varies as $r^{-2}$ for the cylinder and $r^{-3}$ for the sphere. For example, when $r=10$, for a circular cylinder $M \approx 1 \pm 0.01$ whereas for a sphere $M \approx 1 \pm 0.001$.

\section{References}

Aungier R H 1970 A computational method for exact, direct and unified solutions for axisymmetric flow over blunt bodies of arbitrary shape, AFWL-TR-70-16

Back L H, Massier P H \& Gier H L 1965 AIAA J. 31606

Back L H \& Cuffel R F 1971 J. Spacecraft. 8196

Barwell R W \& Davis R M 1971 A computer program for calculating inviscid adiabatic flow about blunt bodies travelling at supersonic and hypersonic speeds at angle of attack, NASA TM X-2334.

Bauer F, Garabedian P R \& Korn D 1975 Supercritical wing $§ 2$ Lecture notes in economics and mathematical systems (New York: Springer Verlag)

Belotserkovskiy O M 1967 Supersonic gas flow around blunt bodies, NASA TT F-453

Boerstoel J W 1974 A transonic hodograph theory for aerofoil design, IMA conference on computational methods and problems in aeronautical flow dynamics, Manchester, NLR Rep. MP $74024 \mathrm{U}$

Boerstoel J W \& Uijlenhoel R R 1970 Lifting aerofoils with supercritical shock-free flow, ICAS paper 70-15, NLR MP 70015U

Chakrabarty S K 1978 AIAA J. 161015.

Chan S T K \& Brashears M R 1974 Finite element analysis of transonic flow, AFFDL-TR-74-11

Chan S T K, Brashears M R \& Young V Y C 1975 Finite element analysis of transonic flow by the method of weighted residuals, AIAA 13th Aerospace Science Meeting, Pasadena, California, AIAA paper 75

Chaplygin S A 1904 On gas flow.Scientific studies at the Moscow University, Physics-mathematica 1 Sciences 21.

Cheng H K and Hafez M M 1976 Lecture notes in physics 47 (ed. H Caleennes) 47101

Cherry T M 1947 Proc. R. Soc. (London) A192 45

Cherry T M 1949a Proc. $R$. Soc. (London) A196 1

Cherry T M 1949b Proc. R. Soc. (London) A196 32

Cherry T M 1950 Proc. R. Soc. A203 551

Cherry T M 1959 J. Aust. Math. Soc. 180

Chushkin N N 1957 Appl. Math. Mech. 21352

Cline M C 1974 AIAA J. 12419 
Crocco L 1965 AIAA J. 3 t824.

Dorodnitsyn A A 1956 On a method of numerical solution of some non-linear problems of aerodynamics, Work of 3rd All Union Mathematical Congress, Part III, (USSR Academy of Sciences) p. 447

Durham F P 1955. The performance characteristics of small rocket-type nozzle, summary report 6. Contract N-123s-82337 T.O.1, Univ. of Colorada, Boulder, Colorado, U.S.A

Emmons H W 1946 The theoretical fiow of a fractionless, adiabatic, perfect gas inside of a twodimensional hyperbolic nozzle, NACA TN-10003.

Emmons H W 1948 Flow of a compressible fluid past a symmetrical airfoil in wind tunnel and in free air, NACA TN-1746.

Frankl F N 1945 Proc. USSR Acad. Sci. 5387

Garabedian P R \& Lieberstein H M 1958 J. Aeronaut Sci. 25109.

Garabedian P R \& Korn D G 1971 On numerical solutions of partial differential equations II (New York: Academic Press)

Goldstein S, Lighthill M J and Craggs J W 1948 Q. J. Mech. Appl. Math. 1344

Gortler H 1939 Z. Angew Math. Mech. 19327

Green J R \& Southwell R V 1944 Philos. Trans. R. Soc. (London) A239 367

Gross M B \& Holt M 1975 in Symposium Transsonicum II IUTAM symposium Gottingen (Berlin: Springer-Verlag) p. 369

Grossman B \& Moretti G 1973 Development of analytical methods of predicting the pressure distribution about a nacelle at transonic speeds-exact solution, NASA CR-112271

Gullstrand T R 1951a The flow over symmetrical aerofoils without incodence in the lower transonic range, Roy. Inst. Technol. (KTH) Tech. Note, AERD 20 Stockholm

Gullstrand T R 1951b The flow over symmetrical aerofoils without incidence at sonic speed, Roy. Inst. Technol. (KTH) Tech. Note AERD 24 Stockholm

Hafez M M, South J \& Murman E M 1979 AIAA J. 17838

Hall I M 1962 Q. J. Mech. Appl. Math. 12487

Hansen $\mathrm{H} 1975$ in Symposium Transonicum II, IUTAM Symposium Cottingen (Eds. by K Oswatitsch \& D Rues) (Berlin: Springer-Verlag) p. 183

Hofez H M \& Cheng H K 1977 ALAA J. 151977

Hofez H M \& Cheng H K 1977 AIAA J. 15786

Holt M 1962 in Symposium Transsonicum, TUTAM Symposium (Berlin: Springer-Verlag) p. 310

Holt M \& Masson B S 1971 Lecture notes in Physics 8243

Imai I 1941 Proc. Phys. Math. Soc. Jpn. 23181

Jameson A 1971 Transonic flow calculation for airfoils and bodies of revolution, Crumman aerodynamics Rept. 391-71-1

Jameson A 1974 Commun. Pure Appl. Math. 27283

Jameson A 1975 Transonic potential flow calculations using conservation form, second AIAA conference on computational fluid dynamics, Hartford

Jameson A 1975 Accelerated iteration sehemes for transonic flow calculations using fast Poisson solves, New York Univ., ERDA Rept. COO-3072-82

Jia Zhan Xue \& Lin Tong Ji 1979 Acta Mech. Sin. 3199

Jia Zhen Xue \& Lin Tong Ji 1981 Transonic flow field in the throat region of axisymmetric nozzles (to be published)

Jones D L \& South Jr. J G 1975 A numerical determination of the bow shock wave in transonic axisymmetric flow about blunt bodies, NASA TM-X.72448

Kliegel J R \& Levine J N 1969 AIAA J. 71375

Klopfer G H \& Holt M 1975 in Symposium Transsonicum II, Symposium Göttingen (Berlin: SpringerVerlag) p. 376

Korn D G 1969 Computation of shock-free transonic flows for airfoil design, NYU Rep. NYU480-125, Courant Inst. Math. Sci.

Krupp J A 1971 The numerical calculation of plane steady transonic flow past thin lifting airfoils, Pep. D. 180-12958-1, Boeing, Seattle, Wash.

Krupp J A \& Murman E M.1972 ALAA J. 10880

Kuo Y H.1948 Two-dimensional irrotational transonic flow, NACA TN-1445

Kuo Y H 1951 Two-dimensional transonic flow airfoils, NACA TN-2356 
Lamb H 1932 Hydrodynamics (New York: Dover) sixth edition p. 70123

Lame G 1834 J. Polytech. Sch. 14191

Laval D 1883 Essentials of fluid dynamics (ed. L Prandt l) (New York: Hafner Publishing Company) p. 268

Lax P D \& Wendroff B 1964 Commun. Pure. Appl. Math. 17381

Lightill M J 1947 Proc. Roy. Soc. (London) A191 323

Lin C C, Reissner E \& Tsien H S 1948 J. Math. Phys. 27220

Lin Tong Ji \& Jia Zhen Xue 1978 Acta Mech. Sin. 11

Lin Tong Ji \& Jia Zhen Xue 1980 Acta Mech. Sin. 4327

Magaus R, Grallaher W \& Yoshihara H 1968 Inviscid supercritical airfoil theory, Trans. Aerodynam AGARD Conf. Proc. No. 35

Magnus R \& Yoshihara H 1970 AIAA J. 82157

Meyer T 1908 On the two-dimensional-forward motion in a gas moving at supersonic speed Research No. 62

Moretti G \& Abbett M 1966 AIAA J. 42136

Moretti G 1969 The choice of a time-dependent technique in gas dynamics, Polytech. Inst. Brookn PIBAL Repl. 69-26, (von Karman Institute for Fluid Dynamics, Lecture Ser. 26 1970)

Moretti G 1970 Transient and asymptotically steady flow of an inviscid, compressible gas past a circle, I. shockless flow, Brooklyn, Polytech. Inst. PIBAL Rep. 70-20 (von Karman Institute for Fluid Dynamics Lecture Ser. 26)

Murman E M \& Cole J D 1971 ALAA J. 9114

Murman E M 1973 Analysis of embedded shock waves calculated by relaxation methods, AIAA computational Fluid Mech. Conf. Palm. (Berlin: Springer-Verlag)

Nieuwland G Y 1967 Transonic potential flow around a family of quasi-elliptical aerofoils section, NLR (Neth) Tech. Rept. T 172

Nixon D 1975 Aeronaut. Q. 2656

Nixon D 1978 AIAA J. 16976

Norrie D H \& de Vries G 1975 in Finite element method in fluid problems (ed. by Oden J T) (University of Alabama Press) p. 21

Norton D L \& Shelton S 1969 Performance of rocket nozzles with low radius of curvature, JPL space programs, summary No. 37-55, Vol. II, Pasadena, Calif, p. 167

Norstrud H 1973 Aeronaut. Q. 24129

Oswatitsch K \& Wieghendt K 1942 Theoretical investigations into the supersteady potential flow at higher velocities (unpublished)

Oswantitsch K 1950 Z. Angew. Math. Mech. 30.17

Reynolds O 1886 Philos. Mag. 21185

Riabouchinsky D 1932 Fluid Mechanics Report (Paris: Academy of Sciences) Vol. 195 p. 398

Rieman B 1851 Foundations of a general theory of functions of a complex variable, Göttingen Mathematical Works, Leipzing, Vol. 187, p. 3

Sauer R 1944 General characteristics of the flow through nozzles at near critical speeds, NACA TM1147

Seria R A 1972 ALAA J. 10603

Shen S F 1975 Finite element method in fluid problems (ed. J. T. Oden), p. 189

Shen S F 1977 Annu. Rev. Fluid Mech. 9440

Simasalsi T 1956 J. Math. Phys. 341

Sobieczky H 1972 Analogue-analytical construction of supercritical profile flow. DGIR-symposium, aircraft aerodynamics at sonic flow

Spreiter J R \& Alksen A Y.1955 Theoretical prediction of pressure distributions on nonlifting air foils at high subsonic speeds, NACA Rept. 1217

Swenson E .V 1968 Commun. Pure. Appl. Math. 21175

Takanashi S 1973 Trans. Jpn Soc. Aeronaut. Space Sci. 1634

Taylor G.I 1930 The flow of air at high speeds past curved surfaces, ARC RM 1831

Tomotika S \& Tamada K 1950 Appl. Math. 7381

Tricomi F 1923 Sulle-equation lineari derivate parziali del secondo ordine, di tipo misto parts 1-7, memori Reale, Nazl. Linoei. Classe Scifis, mat. enat., (Ser. 5) 14, 133247. also see Brown Univ. Transl. A9-T-26 
Tsien H S 1939 J. Aeronaut. Soc. 6399

Tsien H S \& Kuo Y H 1946 Two-dimensional mixed subsonic and supersonic flow of a compressible fluid and the upper critical Mach number, NACA TN 995

Tsien H S 1958 in Fundamentals of gas dynamics (ed. by H W Emmons) (Princeton, New Jersey; Princeton University Press) p. 57

Von Karman 1941 J. Aeronaut. Sci. 8337

Von Karman 1947 J. Math. Phys. 26182

Von Neuman J \& Richtmyer R 1950 J. Appl. Phys. 21232

Von Ringleb F 1940 Z. Angew. Math. Mech. 20 p. 185 